\title{
Arrecifes coralinos de Bocas del Toro, Panamá: II. Distribución, estructura y estado de conservación de los arrecifes de las Islas Bastimentos, Solarte, Carenero y Colón
}

\author{
Héctor M. Guzmán y Carlos A. Guevara \\ Smithsonian Tropical Research Institute, Apartado 2072, Balboa, República de Panamá \\ Fax (507)2280672. Correo electrónico: guzmanh@naos.si.edu
}

Recibido 22-VI-1998. Corregido 16-IX-1998. Aceptado 2-X-1998.

\begin{abstract}
The distribution and conservation status of coral reefs along the northern section of the archipelago of Bocas del Toro are evaluated (ca. $120 \mathrm{~km}$ ), and the structure of 18 reefs around the islands of Bastimentos, Solarte, Carenero and Colón is described. Reef habitats located on the seaward side (exposed) of the islands exhibited the lowest coral cover (8\%) and coral diversity, while leeward reefs (protected) showed the highest living coral cover (32\%) and diversity. Macroalgae coverage was similar in both habitats but high $(50 \%)$ if compared with other continental reefs described in the Ensenada Grande of Almirante Bay (4\%). A total of 54 coral species was recorded, representing a $35 \%$ increase from previous studies for the region and $88 \%$ of the total species described for Panamá. The diversity of reefs, reef organisms and habitats in the archipelago was greater outside the national park of Isla Bastimentos, the only marine protected area in western Panamá. Some recommendations are provided for an integrated management of marine-terrestrial natural resources in the region, in particular, a complete change on the existing limits of the protected area and the creation of new reserves.
\end{abstract}

Key words: Coral reefs, Caribbean, biodiversity, protected area, ecology, reef assessment

La destrucción directa e indirecta de arrecifes coralinos ha sido motivo de preocupación para científicos y autoridades responsables del manejo de recursos naturales, coincidiendo en la falta de protección de dichos ecosistemas como el motivo principal del deterioro en Bocas del Toro y el resto de Panamá. Entre las causas principales de este deterioro se mencionan para el Caribe en general la sobrepesca, la sedimentación y el enriquecimiento (eutrofización) por nutrientes (Rogers 1990, Roberts 1993, 1995). Para Bocas del Toro, la sobrepesca y la sedimentación son amenazas en aumento, que como consecuencia, además de afectar la estructura y funcionamiento del ecosistema arrecifal, podrían tener un impacto socio-económico en toda la región.

Los ecosistemas de la Provincia de Bocas del Toro podrían estar entre los mejores conservados de Panamá. La diversidad biológica tanto marina como terrestre se comenzó a estudiar sistemáticamente a mediados de esta década y coincide con un cambio en el patrón. de desarrollo de la región. Tradicionalmente, el desarrollo de actividades cercanas a la zona costera estuvo limitado a las regiones de Changuinola y Chiriquí Grande, donde las plantaciones de banano y el trasiego de petróleo, respectivamente, fueron 
los ejes principales de la economía regional. Por lo general, el deterioro físico y la contaminación de la zona costera han estado confinados a las áreas de actividad portuaria y a áreas remotas donde los contaminantes (agroquímicos, petróleo y derivados, y plásticos) asociados a estas actividades son transportados por ríos y corrientes (Guzmán y Jiménez 1992, Rodríguez et al. 1993). Actualmente, la pesca tradicional de caracol, tortuga y langosta llevan un curso altamente destructivo y son evidentes las señales de sobrepesca de éstas y otras especies en todo el archipiélago. Desde 1996 el deterioro de los ecosistemas costeros ha aumentado considerablemente con el desarrollo turístico desordenado de la provincia, evidenciandose ya con algunos casos irreversibles.

La Provincia de Bocas del Toro es considerada como una región prioritaria para el desarrollo a nivel nacional, sin embargo, no se conoce de un ordenamiento territorial (marino y terrestre) que permita el uso y manejo racional de los recursos naturales a largo plazo. Por esta razón, se realizarán una serie de cuatro estudios que contemplan la distribución y descripción total de los arrecifes coralinos e inventario de organismos sésiles asociados a estas comunidades, de acuerdo a cuatro unidades geográficas definidas arbitrariamente. Guzmán y Guevara (1998), describen en un primer estudio los arrecifes que bordean la zona litoral continental o de tierra firme desde Punta Valiente hasta la desembocadura del Río Changuinola y además, presentan una introducción más amplia del área de estudio. En dichos arrecifes se encontró una cobertura promedio de coral vivo de $37 \%$ y una diversidad de corales que representa el 53\% del total de especies conocidas para el país (sensu Holst y Guzmán 1993). Esta cobertura de corales podría considerarse la más alta registrada para el Caribe de Panamá hasta el momento y llama la atención el hecho de que proviene de arrecifes donde se esperaría encontrar comunidades afectadas por una alta influencia térrígena.

El presente y segundo estudio comprende la región insular y se describen las características de los arrecifes de las islas de Bastimentos,
Solarte, Carenero y Colón. En particular, se evalúa el estado del Parque Nacional Marino Isla Bastimentos, única área protegida del $\mathrm{Ca}$ ribe occidental de Panamá. Dicha área fue creada en setiembre de 1988 (Mou-Sue 1993) siguiendo los delineamientos propuestos por Alvarado (1987).

\section{MATERIALES Y MÉTODOS}

Área de estudio: La Provincia de Bocas del Toro se encuentra localizada al occidente de la República de Panamá entre los $8^{\circ} 30^{\prime}$ y $9^{\circ} 40^{\prime}$ de latitud norte y $82^{\circ} 56^{\prime}$ y los $81^{\circ} 8^{\prime}$ de longitud oeste; limita al norte con el Mar Caribe, al sur con la Provincia de Chiriquí, al este con la Provincia de Veraguas y al oeste con la República de Costa Rica. La provincia tiene una superficie de ca. $8917 \mathrm{~km}^{2}$ (Rodríguez et al. 1993). Los mayores centros poblados de la región insular y del área de estudio están localizados en las Islas Bastimentos, Colón y Carenero. La precipitación es algo menor (2 000$3000 \mathrm{~mm} / \mathrm{año}$ ) en la zona costera continental desde Changuinola hasta la parte noroeste de la Laguna de Chiriquí y con influencia sobre las islas de Cristóbal y el norte de Isla Colón. Mientras que la precipitación es mayor (3 000$4000 \mathrm{~mm} / \mathrm{año}$ ) en el sistema insular del archipiélago, el área del presente estudio (Anónimo 1988a).

Reconocimiento de arrecifes y muestreo: Nuestro estudio comprende tres aspectos básicos para la evaluación de los arrecifes de coral: (1) distribución de los arrecifes coralinos; (2) inventario sobre la diversidad de corales y algunos organismos principales asociados al arrecife; (3) zonación, cobertura y abundancia relativa de corales y principales organismos sésiles en los arrecifes de la región; y (4) abundancia y diversidad de corales juveniles o nuevos reclutas. Además, se describe brevemente el estado de conservación de los arrecifes y otras comunidades litorales, incluyendo los bosques de manglar y el bosque lluvioso tropical. Esto permitirá evaluar 
el estado actual de las comunidades de la zona costera, con particular énfasis en los arrecifes coralinos y proyectar así las áreas naturales vulnerables a cambios ambientales, naturales o antrópicos.

Para cada arrecife estudiado se realizaron dos tipos de evaluaciones que permitiría la caracterización adecuada de la estructura de la comunidad arrecifal (adaptado de Sullivan y Chiappone 1992, Sullivan et al. 1994). Guzmán y Guevara (1998), explican detalladamente como se evaluaron los arrecifes, incluyendo la taxonomía de especies y la caracterización de los arrecifes por medio de transectos. En cada transecto, se estimó visualmente la cobertura de cada organismo sésil presente empleando una cuadrícula de PVC de $1 \mathrm{~m}^{2}$, subdividida en 100 celdas de $100 \mathrm{~cm}^{2}$ cada una (total de $45 \mathrm{~m}^{2}$ por arrecife). Además, se estimó el número de individuos juveniles o reclutas $/ \mathrm{m}^{2}$ de especies de coral presentes en los transectos, tomando en cuenta únicamente los individuos menores a $4 \mathrm{~cm}$ producto de reproducción sexual (sensu Rylaarsdam 1983, Rogers et al. 1984). La nu- meración asignada a los arrecifes, comenzando por el 25, corresponde a la base de datos del Instituto Smithsonian de Investigaciones Tropicales, las cuales contiene otros arrecifes descritos anteriormente para la región (ver Guzmán y Guevara 1998).

\section{RESULTADOS}

Se presenta la distribución de arrecifes coralinos para cada una de las cuatro islas estudiadas (Fig. 1) y se describen un total de 18 arrecifes coralinos para un total de $120 \mathrm{~km}^{2} \mathrm{de}$ costa evaluados (Cuadro 1). Se estimó una distancia mínima de separación entre los arrecifes descritos de ca. $3 \mathrm{~km}$ (donde existen estos), de forma tal que el muestreo fuera representativo de la diversidad de hábitats y condiciones orográficas de la zona costera y no de una isla en particular. Los resultados se presentan analizando toda el área de estudio en su conjunto y posteriormente se presentan en forma individual para cada isla estudiada.

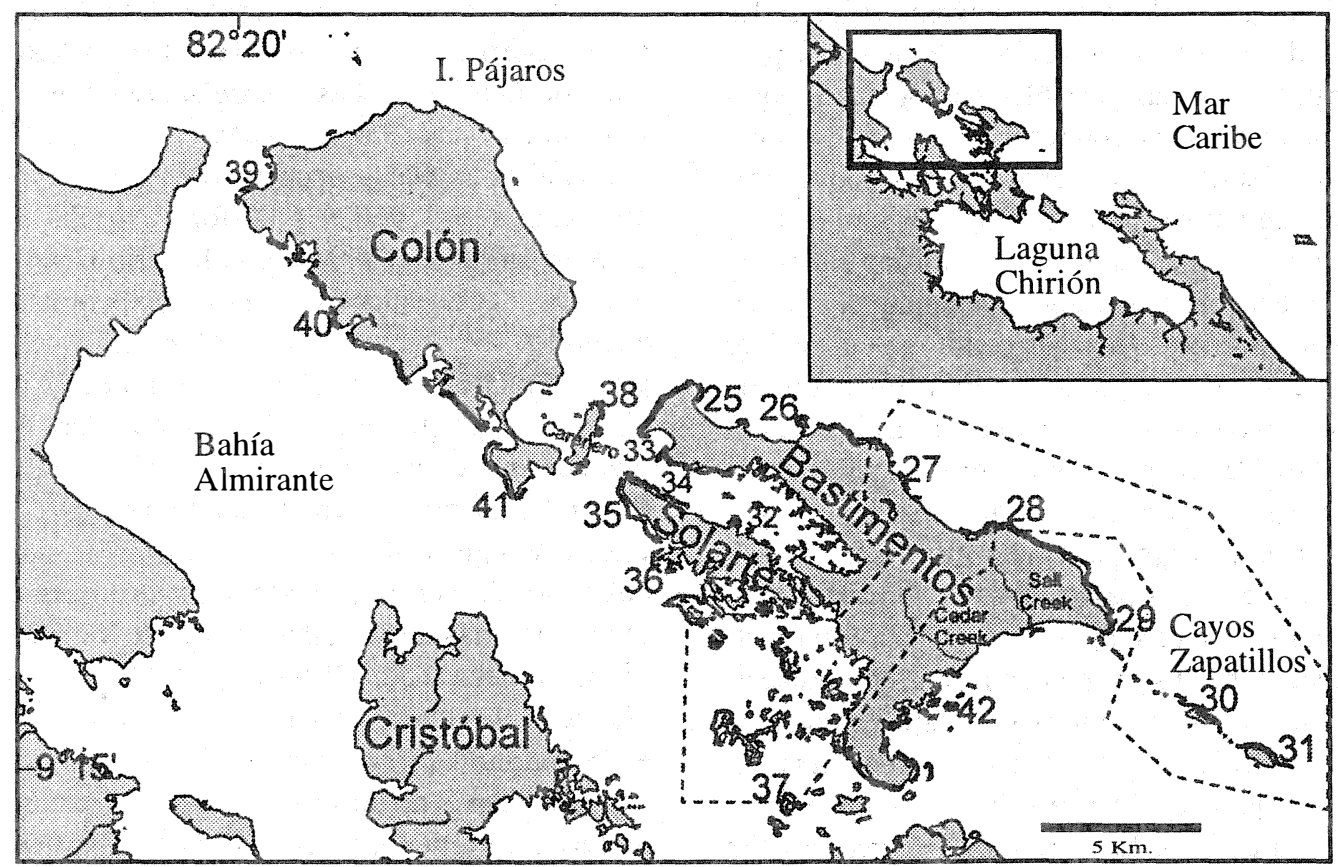

Fig. 1. Bocas del Toro y las principales áreas de estudio; Islas Bastimentos, Solarte, Carenero y Colón. La ubicación geográfica de los 18 arrecifes descritos corresponde al Cuadro 1. La línea solida de trazado negro representa la distribución aproximada de los arrecifes y el área demarcada corresponde al Parque Nacional Marino Isla Bastimentos (PNMIB). 


\section{CUADRO 1}

Localización de los 18 arrecifes coralinos estudiados en las Islas Colón, Carenero, Bastimentos y Solarte, Bocas del Toro, Panamá (ver Fig. 1) y la profundidad máxima de desarrollo arrecifal (metros)

$\mathrm{N}^{\mathbf{o}}$

25

26

27

28

29

30

31

32

33

35

36

37

38

39

40

41

42

Latitud/Longitud
$9^{\circ} 21^{\prime} 23^{\prime \prime} \mathrm{N} / 82^{\circ} 11^{\prime} 54^{\prime \prime} \mathrm{W}$
$9^{\circ} 20^{\prime} 52^{\prime \prime} \mathrm{N} / 82^{\circ} 10^{\prime} 09^{\prime \prime} \mathrm{W}$
$9^{\circ} 20^{\prime} 24^{\prime \prime} \mathrm{N} / 82^{\circ} 08^{\prime} 33^{\prime \prime} \mathrm{W}$
$9^{\circ} 19^{\prime} 11^{\prime \prime} \mathrm{N} / 82^{\circ} 06^{\prime} 26^{\prime \prime} \mathrm{W}$
$9^{\circ} 17^{\prime} 35^{\prime \prime} \mathrm{N} / 82^{\circ} 04^{\prime} 51^{\prime \prime} \mathrm{W}$
$9^{\circ} 16^{\prime} 07^{\prime \prime} \mathrm{N} / 82^{\circ} 03^{\prime} 24^{\prime \prime} \mathrm{W}$
$9^{\circ} 15^{\prime} 07^{\prime \prime N} \mathrm{~N} / 82^{\circ} 01^{\prime} 45^{\prime \prime} \mathrm{W}$
$9^{\circ} 19^{\prime} 38^{\prime \prime} \mathrm{N} / 82^{\circ} 11^{\prime} 31^{\prime \prime} \mathrm{W}$
$9^{\circ} 20^{\prime} 14^{\prime \prime N} / 82^{\circ} 12^{\prime} 29^{\prime \prime} \mathrm{W}$
$9^{\circ} 19^{\prime} 51^{\prime \prime N} / 82^{\circ} 12^{\prime} 52^{\prime \prime} \mathrm{W}$
$9^{\circ} 19^{\prime} 33^{\prime \prime N} / 82^{\circ} 13^{\prime} 16^{\prime \prime} \mathrm{W}$
$9^{\circ} 18^{\prime} 30^{\prime \prime} \mathrm{N} / 82^{\circ} 12^{\prime} 37^{\prime \prime} \mathrm{W}$
$9^{\circ} 14^{\prime} 21^{\prime \prime} \mathrm{N} / 82^{\circ} 10^{\prime} 23^{\prime \prime} \mathrm{W}$
$9^{\circ} 21^{\prime} 19^{\prime \prime N} / 82^{\circ} 13^{\prime} 37^{\prime \prime} \mathrm{W}$
$9^{\circ} 17^{\prime} 46^{\prime \prime} \mathrm{N} / 82^{\circ} 16^{\prime} 04^{\prime \prime} \mathrm{W}$
$9^{\circ} 22^{\prime} 39^{\prime \prime} \mathrm{N} / 82^{\circ} 18^{\prime} 17^{\prime \prime} \mathrm{W}$
$9^{\circ} 19^{\prime} 44^{\prime \prime} \mathrm{N} / 82^{\circ} 15^{\prime} 14^{\prime \prime} \mathrm{W}$
$9^{\circ} 16^{\prime} 00^{\prime \prime} \mathrm{N} / 82^{\circ} 07^{\prime} 13^{\prime \prime} \mathrm{W}$

La estructura (diversidad, abundancia y zonación), de los 18 arrecifes descritos se separó estadísticamente en dos grandes grupos (hábitats), de acuerdo a su ubicación en el archipiélago; barlovento y sotavento (Fig. 2). En dos de cuatro combinaciones realizadas para agrupar distintas variables ecológicas (cobertura, diversidad), con respecto a distintos organismos sésiles, se encontró que los nueve arrecifes de hábitat protegidos (32-37, 40-42) del sistema insular (sotavento) son similares en composición de especies y abundancia de corales y diferentes a los arrecifes expuestos (ver Fig. 2A). Más importante aún, la estructura de estos arrecifes, basada en la estimación de cobertura de los principales grupos sésiles que conforman el arrecife (corales, esponjas y macroalgas), se agrupó de igual forma (Fig. 2B). En los restantes casos, donde se utilizó la cobertura de macroalgas y esponjas individualmente para observar la similitud entre arrecifes, no se observó un patrón claro y similar al anterior (Fig. 2C y D), lo cual demuestra la importancia de evaluar múltiples organismos sésiles al estudiar la estructura de arrecifes. Sin embargo, el dendrograma basado en cobertura de macroalgas a pesar de no reflejar la estructura arrecifal, separó siete de los nueve arreci-

\section{Localidad}

m

Punta Toro
Cayo Wild Cane
Playa Larga Oeste
Fish Hole
Old Point
Cayo Zapatilla Oeste
Cayo Zapatilla Este
Ensenada Hospital (Parche)
Punta Juan Brown
Cayo Solarte Norte
Cayo Solarte Sur
Cayo Buttonwood Oeste
Cayo Adriana Este
Cayo Carenero Norte
Lime Point
Conch Point
Mangrove Point
Isla Bastimento Este (Parche)

fes expuestos por presentar coberturas superiores al 55-60\% (Fig. 2D, Cuadro 2).

El promedio global de cobertura de coral vivo para los arrecifes protegidos fue de ca. $30 \%$, comparado con $8 \%$ en arrecifes expuestos (Cuadro 2). El grupo de las macroalgas, que podría reflejar el estado de salud del ecosistema en estas islas es bastante alto ( $>30 \%)$ para ambos hábitats, pero superior en los arrecifes expuestos (ca. 70\%). De forma similar se comportan las algas rojas coralinas, mientras que los demás grupos sésiles (octocorales, zoántidos, esponjas), no presentan una variación amplia entre arrecifes. Las esponjas son el segundo grupo animal de major importancia, con una cobertura de ca. 5\% (Cuadro 2). Sin embargo, no se encontraron diferencias estadísticas significativas entre los promedios de cobertura de corales, macroalgas, esponjas y algas coralinas de arrecifes expuestos y protegidos (Prueba Mann-Whitney, $\mathrm{p}>0.05$ ), relacionado esto con la alta variabilidad observada en cobertura para cada arrecife individual.

En cuanto al desarrollo de la estructura arrecifal, se observa un basamento con sustrato solido (>95\%) y menos arenoso que se extiende hasta una baja profundidad en los arrecifes a barlovente, el cual se forma desde la 

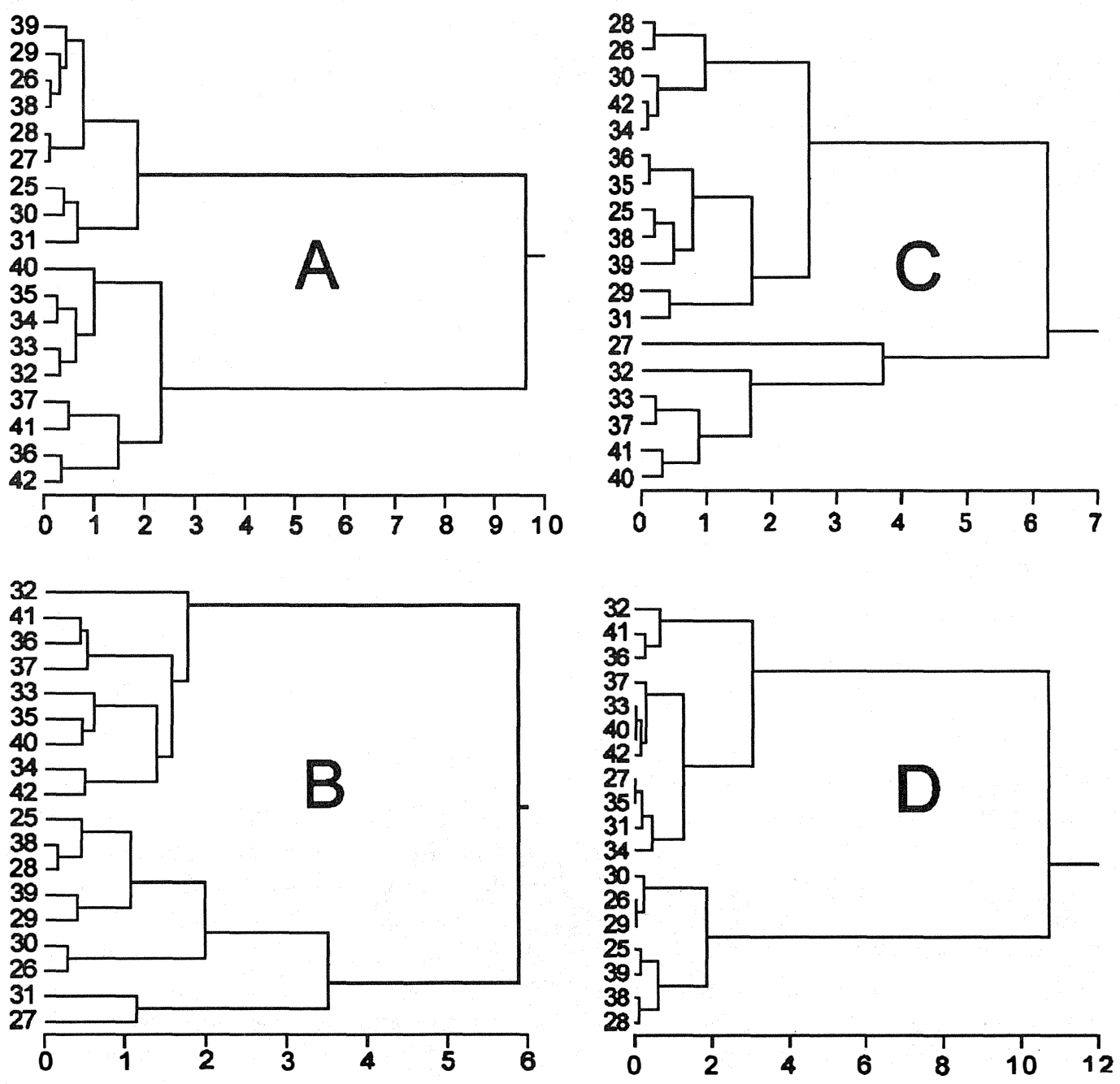

Distancia

Fig. 2. Análisis de grupos comparando los diferentes táxones dentro de las variables estudiadas en los 18 arrecifes. A) cobertura y diversidad de corales escleractínios; B) estructura de los arrecifes basado en cobertura de corales escleractínios, macroalgas, esponjas y algas rojas coralinas; C) cobertura y diversidad de esponjas y D) cobertura de macroalgas. Los dendrogramas se basan en distancia euclidiana, amalgamados por el método de Ward.

cresta del arrecife hasta su base (ca. $15 \mathrm{~m}$ ) y posiblemente cementado por la alta abundancia de algas rojas coralinas (ver Cuadros 1 y 2 ). Además, se observan extensas plataformas arrecifales profundas (ca. 20-30 m) aisladas del sistema insular y hacia el norte del archipiélago (dichas estructuras no fueron descritas en este estudio). Los arrecifes internos o protegidos presentan estructuras desarrolladas hasta una mayor prof undidad (ca. 20-25 m), pero por lo general entremezclados con parches de arena.

La distribución vertical de los principales grupos sésiles estudiados indica que los corales en arrecifes protegidos tienen una cobertura mayor y estadísticamente significativa a profundidades superiores a los $5 \mathrm{~m}$ y una menor cobertura en ambientes someros (ANOVA 


\section{CUADRO 2}

Porcentaje promedio (error estándar) total de cobertura de coral vivo y principales grupos taxonómicos por $m^{2}$ (esponjas, algas, zoántidos), para cada uno de los 18 arrecifes estudiados en Bocas del Toro, Panamá (según Cuadro 1) Cobertura promedio para arrecifes expuestos $(E X)$, arrecifes protegidos $(P R)$ y total (TO)

$\begin{array}{ccccccccc}\text { Sitio Escleractínio } & \text { Octocoral } & \text { Hidrocoral } & \text { Esponja } & \text { Zoántido } & \text { Macroalga } & \text { Alga Coralina } & \text { Arena } \\ 25 & 15.7(1.5) & 0.3(0.1 & 0.7(0.2) & 2.0(0.6) & 2.8(0.5) & 76.8(1.5) & 1.6(0.5) \\ 26 & 4.7(0.9) & 0.1(0.0) & 0.5(0.2) & 0.5(0.1) & 0.6(0.3) & 67.4(3.0) & 23.2(3.2) & 3.0(0.8) \\ 27 & 8.5(1.4) & 0.1(0.1) & 1.5(0.4) & 18.5(4.3) & 1.8(0.5) & 42.3(4.4) & 27.1(3.7) & 0.1(0.1) \\ 28 & 7.4(0.9) & 0.1(0.0) & 0.3(0.1) & 0.7(0.2) & 0.0(0.0) & 88.4(0.9) & 2.9(0.8) & 0.1(0.1) \\ 29 & 7.9(1.3) & 0.1(0.1) & 2.9(0.7) & 4.8(2.3) & 2.9(0.8) & 68.0(2.7) & 13.3(2.3) & - \\ 30 & 12.3(1.1) & - & 2.5(0.9) & 0.1(0.1) & 0.4(0.2) & 63.5(3.3) & 21.2(3.4) & - \\ 31 & 7.9(1.6) & 1.4(0.4) & 2.0(0.4) & 7.7(2.1) & 4.3(1.7) & 45.7(3.3) & 30.9(3.9) & - \\ 32 & 24.9(3.0) & 0.3(0.1) & 0.6(0.2) & 12.7(1.8) & 2.2(0.5) & 7.1(1.2) & 0.4(0.2) & 51.8(2.7) \\ 33 & 27.5(3.3) & 0.7(0.2) & 0.4(0.1) & 6.8(0.8) & 2.6(2.1) & 35.0(4.0) & 0.3(0.2) & 26.8(4.1) \\ 34 & 29.0(4.2) & 0.7(0.1) & 0.5(0.2) & 0.8(0.2) & 0.2(0.1) & 51.6(5.1) & 12.9(2.5) & 4.3(1.8) \\ 35 & 27.7(4.5) & 1.0(0.2) & 0.5(0.3) & 2.7(0.4) & 2.2(2.2) & 42.5(4.7) & 3.8(1.6) & 19.6(3.6) \\ 36 & 37.3(4.7) & 0.2(0.1) & - & 2.3(0.3) & - & 23.6(2.6) & 4.1(1.3) & 32.5(5.5) \\ 37 & 43.9(4.1) & 0.0(0.0) & 0.2(0.2) & 6.1(0.7) & 2.0(0.6) & 30.6(4.1) & 0.5(0.5) & 16.7(2.9) \\ 38 & 6.4(1.0) & 0.2(0.1) & 1.2(0.3) & 2.2(0.4) & 0.5(0.2) & 86.2(1.4) & 2.9(0.8) & 0.4(0.2) \\ 39 & 1.2(0.3) & 1.8(0.4) & 0.8(0.3) & 4.9(0.7) & 1.6(0.9) & 80.2(3.1) & 8.7(3.3) & 0.9(0.4) \\ 40 & 14.6(2.3) & 3.3(0.9) & 1.0(0.4) & 2.5(0.5) & 1.2(0.7) & 35.3(3.6) & 5.6(1.9) & 36.5(4.3) \\ 41 & 48.9(4.4) & 0.1(0.0) & 0.2(0.1) & 3.6(0.5) & - & 17.4(2.9) & 6.4(1.6) & 23.5(4.3) \\ 42 & 37.4(3.9) & 0.4(0.2) & 4.3(1.3) & 0.2(0.1) & 0.4(0.3) & 37.8(3.8) & 18.9(3.0) & 0.7(0.3) \\ \text { EX } & 8 & 0.3 & 1.4 & 4.6 & 1.7 & 68.7 & 14.6 & 0.9 \\ \text { PR } & 32.5 & 0.6 & 1 & 4.2 & 1.5 & 31.2 & 5.9 & 23.6 \\ \text { TO } & 20.2 & 0.6 & 1.2 & 4.4 & 1.6 & 50 & 10.3 & 15.5 \\ & & & & & & & \end{array}$

Kruskal-Wallis, p<0.001), mientras que se observó una abundancia similar (Kruskal-Wallis $\mathrm{p}>0.05$ ) entre profundidades de arrecifes expuestos (Figs. 3 y 4). Las macroalgas presentan una zonación similar en los arrecifes expuestos, mientras que en los protegidos la abundancia es más variable pero con tendencia a ser menos abundantes en aguas de profundidad intermedia. Sin embargo, las diferencias encontradas para ambos hábitats no son significativas (Kruskal-Wallis, p>0.05). Las esponjas presentan un patrón de zonación bastante uniforme (Kruskal-Wallis, p $>0.05$ para ambos hábitats), con algunas excepciones como los arrecifes 27 y 32 con coberturas superiores al $10-20 \%$ hasta profundidades intermedias (Fig. 3, Cuadro 2). En particular, los arrecifes a barlovento (expuestos), están cubiertos por la esponja incrustante Cliona spp., la cual ha competido agresivamente con los corales Porites astreoides, Diploria clivosa y D. strigosa. Las algas rojas coralinas presentan una disminución en la cobertura al aumentar la profundidad de los arrecifes expuestos (Kruskal-Wallis, $\mathrm{p}=0.08$ ) y una distribución similar en arrecifes protegidos (Kruskal-Wallis, p>0.05) (Figs. $3 \mathrm{y}$ 4). Una vez más, la alta variabilidad de los promedios de coberturas de organismos sésiles entre arrecifes dentro de un mismo hábitat, impide encontrar diferencias estadísticas más claras.

La cobertura-composición de las especies de corales cambió notablemente con la profundidad y el tipo de hábitat. Por un lado, en los hábitats expuestos y someros son más abundantes las especies Diploria clivosa, Porites astreoides y Millepora complanata, mientras que a mayor profundidad dominan especies como Agaricia tenuifolia, Siderastrea siderea, y A. undata (Fig. 5). El zoántido Palythoa caribaeorum está ampliamente distribuido y se encuentra más abundante a poca profundidad. El coral Acropora palmata $(0.23-0.81 \%)$ y A. cervicornis $(<0.05 \%)$, se encontraron en baja abundancia en áreas expuestas y hasta $5 \mathrm{~m}$ de profundidad únicamente. Por otro lado, en hábitats protegidos el coral ramificado Porites furcata domina ampliamente los arrecifes someros e intermedios, pero Agaricia tenuifolia es la especie más abundante en aguas intermedias y profundas (Fig. 6). Los corales masivos Porites astreoides, Col- 

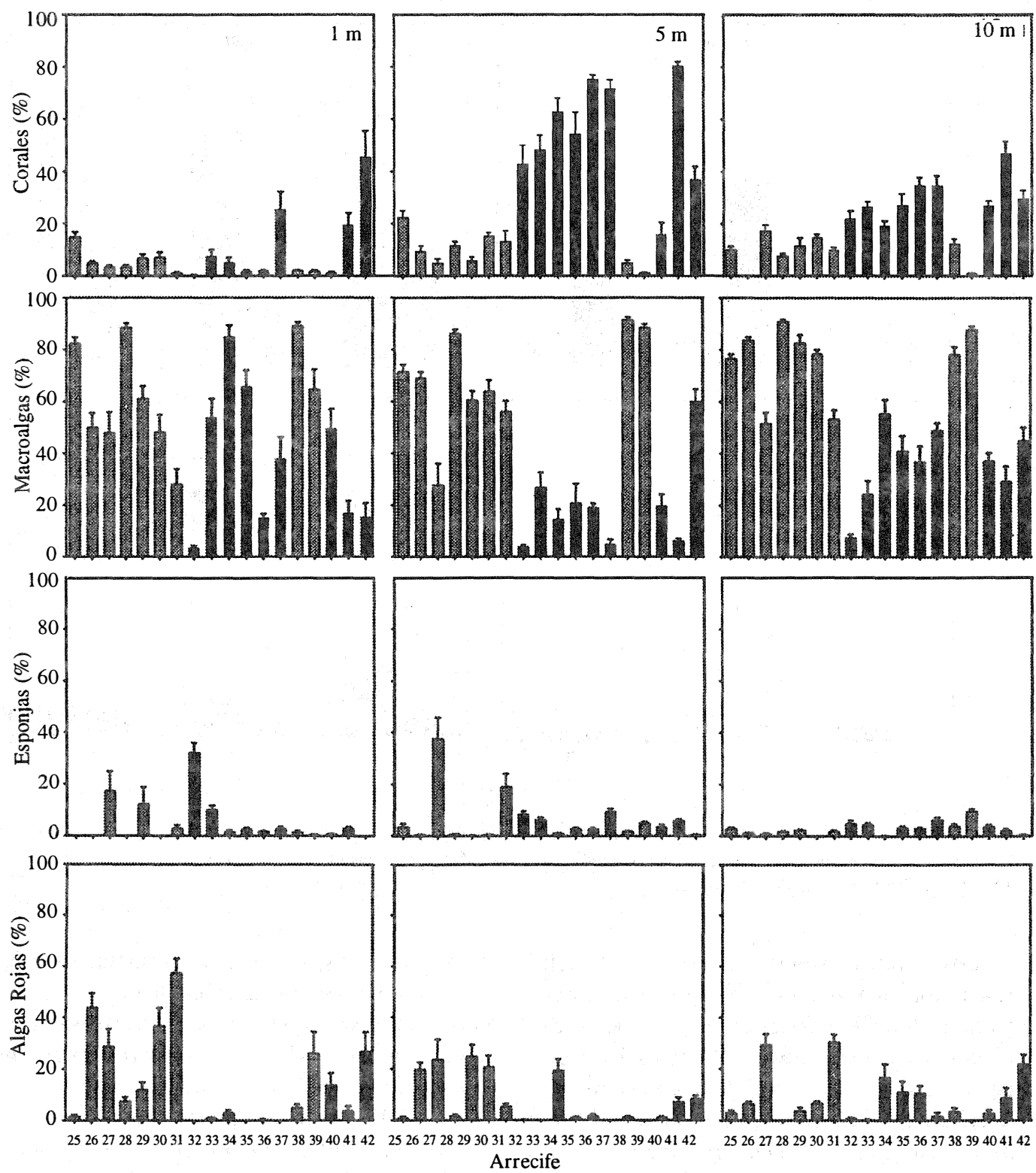

Fig. 3. Porcentaje promedio y error estándar para la cobertura relativa de coral vivo escleractínio, esponjas, macroalgas y algas rojas coralinas por $\mathrm{m}^{2}$, para cada profundidad indicada y arrecife individual descrito en Bocas del Toro, Panamá (según Fig. 1). Números en eje inferior indican los 18 arrecifes estudiados (ver Cuadro 1), clasificados como expuestos (barras grises) y protegidos (barras negras).

pophyllia natans y Montastraea franksi son más abundantes hacia aguas intermedias y profundas, con la excepción de Siderastrea siderea que se distribuye de forma similar por todo el arrecife. Solenastrea hyades y Madracis spp. son más abundante hacia la base de los arrecifes
(Fig. 6). Acropora palmata presentó una abundancia alta en aguas someras (4.8\%) y baja en aguas más profundas $(0.1 \%)$, mientras que $A$. cervicornis es más común en aguas intermedias y profundas $(0.83 \%$ y $0.66 \%$ respectivamente) y menos en hábitats someros $(0.1 \%)$. 


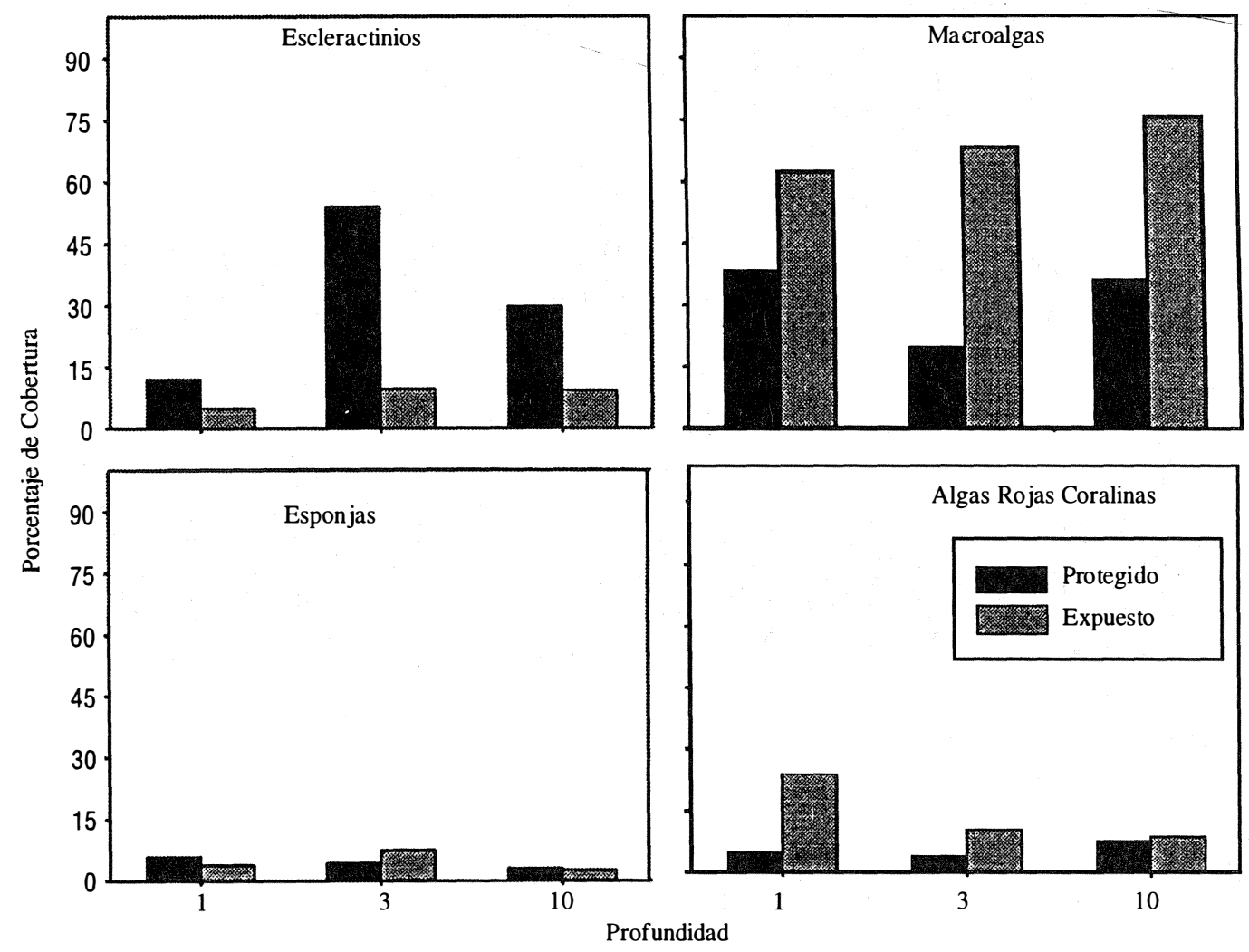

Fig. 4. Resumen de porcentaje promedio para la cobertura relativa de coral vivo escleractínios, esponjas, macroalgas y algas rojas coralinas por $\mathrm{m}^{2}$, para cada profundidad indicada y arrecifes agrupados en expuestos (gris) y protegidos (negro), en Bocas del Toro, Panamá (según Fig. 2).

Los corales juveniles (nuevos reclutas), presentaron una abundancia opuesta a la encontrada al medirse cobertura general de especies con respecto al tipo de hábitat, es decir, un mayor número promedio de reclutas $/ \mathrm{m}^{2}$ en arrecifes expuestos $(0.58 \pm 0.19)$ que en arrecifes protegidos $(0.39 \pm 0.11)$. Sin embargo, dicha diferencia no fue estadísticamente significativa (Mann-Whitney, $\mathrm{p}>0.05$ ). En arrecifes expuestos los reclutas presentaron una distribución mayor en aguas someras e intermedias $(0.77$ individuos $/ \mathrm{m}^{2}$ ), comparado con 0.2 indivi$\mathrm{duos} / \mathrm{m}^{2}$ en aguas profundas. El patrón de reclutamiento en hábitats protegidos fue lo contrario; 0.61 individuos $/ \mathrm{m}^{2}$ en la parte profunda del arrecife, comparado: con 0.27 individuos $/ \mathrm{m}^{2}$ para aguas someras e intermedias. No se encontraron individuos juveniles de Acropo- ra cervicornis y A. palmata en ningún arrecife expuesto o protegido. La abundancia por especies de reclutas $/ \mathrm{m}^{2}$ es similar para arrecifes expuestos y protegidos; Porites astreoides $>\mathrm{Fa}$ via fragum $>$ Siderastrea siderea $>$ Agaricia tenuifolia=A. Agaricites (Cuadro 3).

La diversidad total de corales escleractínios fue de 54 especies, de las cuales se encontraron 47 y 35 especies para arrecifes protegidos y expuestos, respectivamente (Cuadros 4 y 5). Los octocorales son poco diversos en estos arrecifes, encontrándose un total de 25 especies y poca diferencia entre arrecifes protegidos con 22 especies y 17 en arrecifes expuestos (Cuadros 4 y 6). Las esponjas resultaron ser un grupo bastante diverso dentro del área de estudio, con un total de 37 especies, todas representadas en los arrecifes protegidos y solamente 29 


\section{CUADRO 3}

Porcentaje (error estándar) de reclutas por $m^{2}$ para las especie de corales escleractínios encontrados en los 18 arrecifes estudiados de Bocas del Toro, Panamá. Solamente especies con porcentaje mayor a $0.01 \%$

\begin{tabular}{|c|c|c|c|c|c|c|c|c|c|c|c|c|c|c|c|c|c|c|}
\hline Especies & 25 & 26 & 27 & 28 & 29 & 30 & 31 & 32 & 33 & 34 & 35 & 36 & 37 & 38 & 39 & 40 & 41 & 42 \\
\hline Agaricia agaricites & - & $\begin{array}{c}0.04 \\
(0.04)\end{array}$ & $\begin{array}{c}0.16 \\
(0.16)\end{array}$ & - & $\begin{array}{r}0.09 \\
(0.07\end{array}$ & $\begin{array}{c}0.11 \\
(0.08)\end{array}$ & - & - & - & - & - & - & - & - & $\begin{array}{c}0.04 \\
(0.04)\end{array}$ & - & - & - \\
\hline Agaricia danai & - & - & - & - & - & $\begin{array}{l}0.07 \\
(0.07)\end{array}$ & - & - & $\begin{array}{c}0.09 \\
(0.09)\end{array}$ & - & - & - & - & - & - & - & - & - \\
\hline Agaricia fragilis & - & - & - & - & - & - & - & - & - & $\begin{array}{c}0.04 \\
(0.04)\end{array}$ & - & - & - & - & - & - & - & - \\
\hline Agaricia lamarcki & - & - & - & - & - & - & - & - & - & - & - & $\begin{array}{c}0.02 \\
(0.02)\end{array}$ & - & - & - & - & - & - \\
\hline Agaricia tenuifolia & - & $\begin{array}{c}0.07 \\
(0.07)\end{array}$ & - & - & - & $\begin{array}{l}0.29 \\
(0.17)\end{array}$ & - & - & - & $\begin{array}{c}0.07 \\
(0.07)\end{array}$ & - & - & $\begin{array}{c}0.22 \\
(0.16)\end{array}$ & - & - & - & - & $\begin{array}{c}0.29 \\
(0.18)\end{array}$ \\
\hline Diploria strigosa & $\begin{array}{c}0.07 \\
(0.05)\end{array}$ & - & - & - & - & - & - & - & - & - & - & - & - & - & - & - & - & - \\
\hline Favia fragum & - & $\begin{array}{c}0.42 \\
(0.17)\end{array}$ & $\begin{array}{c}0.31 \\
(0.31)\end{array}$ & $\begin{array}{c}0.20 \\
(0.11)\end{array}$ & $\begin{array}{l}0.04 \\
0.04\end{array}$ & $\begin{array}{l}0.16 \\
(0.11\end{array}$ & $\begin{array}{c}0.11 \\
(0.11)\end{array}$ & - & $\begin{array}{l}0.07 \\
(0.07)\end{array}$ & - & $\begin{array}{c}0.07 \\
(0.05)\end{array}$ & - & - & $\begin{array}{c}0.53 \\
(0.22)\end{array}$ & - & $\begin{array}{c}0.11 \\
(0.08)\end{array}$ & - & - \\
\hline Leptoseris cucullata & - & - & - & - & - & $\begin{array}{c}0.09 \\
(0.07)\end{array}$ & - & - & - & - & $\begin{array}{c}0.07 \\
(0.07)\end{array}$ & - & $\begin{array}{c}0.11 \\
(0.08)\end{array}$ & - & - & - & - & $\begin{array}{c}0.22 \\
(0.11)\end{array}$ \\
\hline Manicina areolata & - & - & - & - & - & - & - & - & - & - & - & $\begin{array}{c}0.22 \\
(0.11)\end{array}$ & - & - & - & - & - & - \\
\hline Millepora alcicornis & - & - & - & - & - & - & - & - & - & - & $\begin{array}{c}0.04 \\
(0.04)\end{array}$ & - & - & - & - & - & - & - \\
\hline Porites colonensis & - & - & - & - & - & - & - & - & - & - & - & - & - & - & - & - & - & $\begin{array}{l}0.02 \\
(0.02\end{array}$ \\
\hline Porites furcata & - & - & - & $\begin{array}{c}0.07 \\
(0.07)\end{array}$ & $\cdots$ & $\begin{array}{c}0.07 \\
(0.07)\end{array}$ & - & - & - & - & - & $\begin{array}{c}0.13 \\
(0.09)\end{array}$ & - & - & - & - & - & - \\
\hline Siderastrea radians & - & - & - & - & - & - & - & - & $\begin{array}{l}0.07 \\
(0.07\end{array}$ & - & - & $\begin{array}{c}0.04 \\
(0.04)\end{array}$ & - & - & - & - & - & - \\
\hline Siderastrea siderea & $\begin{array}{c}0.36 \\
(0.13)\end{array}$ & $\begin{array}{c}0.07 \\
(0.07)\end{array}$ & $\begin{array}{c}0.02 \\
(0.02)\end{array}$ & $\begin{array}{l}0.07 \\
(0.07\end{array}$ & $\begin{array}{c}0.04 \\
(0.04)\end{array}$ & - & - & $\begin{array}{c}0.07 \\
(0.05)\end{array}$ & $\begin{array}{c}0.07 \\
(0.05)\end{array}$ & $\begin{array}{c}0.04 \\
(0.04)\end{array}$ & $\begin{array}{c}0.02 \\
(0.02)\end{array}$ & - & - & $\begin{array}{c}0.04 \\
(0.04)\end{array}$ & - & - & - & $\begin{array}{c}0.09 \\
(0.09)\end{array}$ \\
\hline Solenastrea hyades & - & - & - & - & - & - & - & - & - & - & - & $\begin{array}{c}0.04 \\
(0.04)\end{array}$ & - & - & - & $\begin{array}{c}0.02 \\
(0.02)\end{array}$ & - & - \\
\hline $\begin{array}{l}\text { Stephanocoenia } \\
\text { intercepta }\end{array}$ & - & - & - & - & - & - & - & $\begin{array}{c}0.01 \\
(0.01)\end{array}$ & $\begin{array}{c}0.16 \\
(0.09)\end{array}$ & - & - & - & - & - & - & - & - & - \\
\hline Total Reclutas & 0.67 & 0.91 & 0.73 & 0.47 & 0.41 & 1.1 & 0.15 & 0.09 & 0.53 & 0.31 & 0.31 & 0.54 & 0.46 & 0.75 & 0.06 & 0.21 & 0 & 0.95 \\
\hline
\end{tabular}



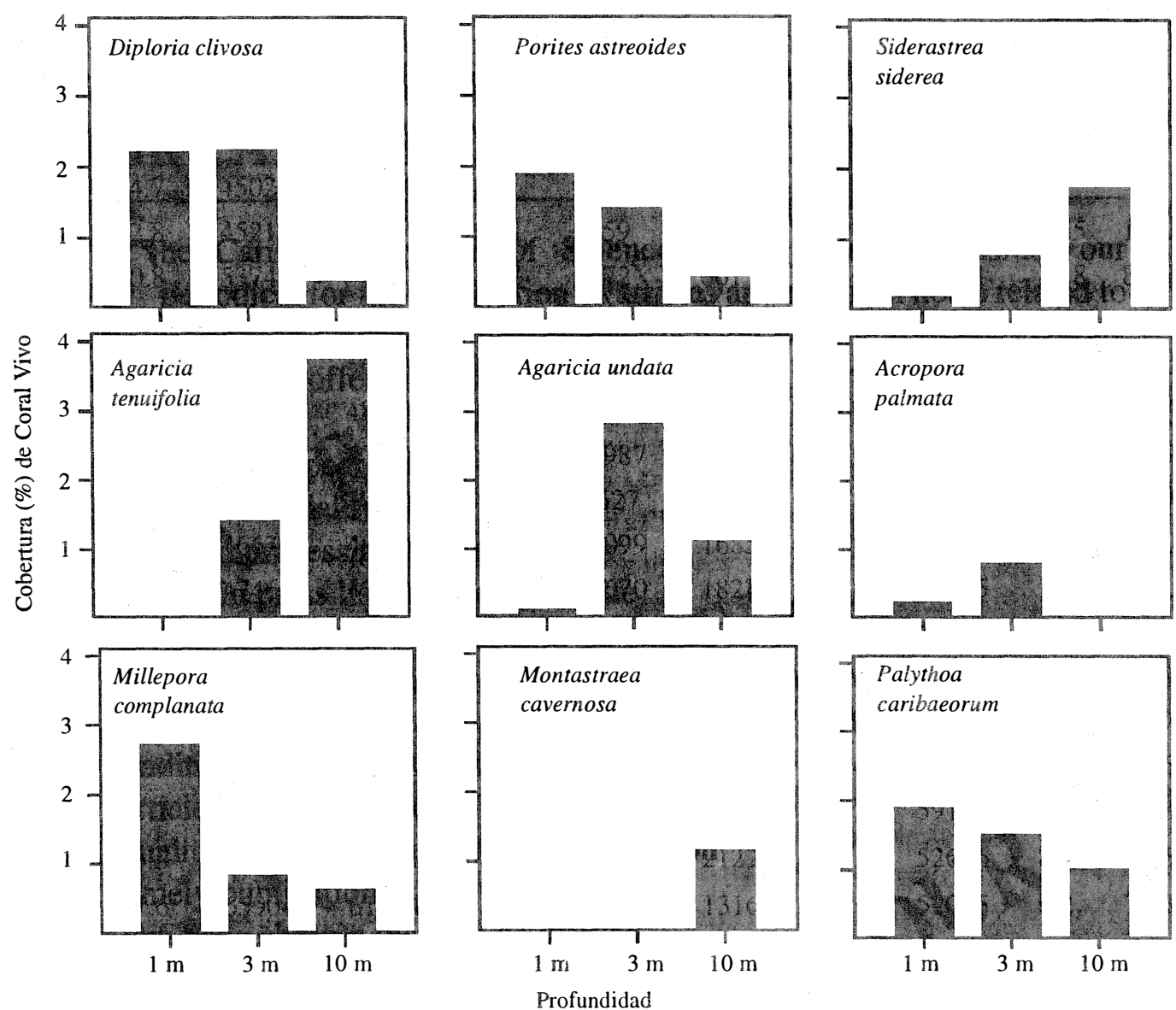

Fig. 5. Cobertura (\%) absoluta de coral vivo para todos los arrecifes agrupados como expuestos (según Fig. 2), para las especies más abundantes de coral y otros táxones encontradas en los transectos y para las tres profundidades (zonación). Se incluyen únicamente las especies con porcentajes mayores al $0.5 \%$.

especies en los arrecifes expuestas (Cuadros 4 y 7). Además, se encontraron seis especies de anémonas y dos especies de coral negro y zoántidos, distribuídos en ambos hábitats de forma similar (Cuadro 6).

Es importante notar que la composición de los sedimentos en las áreas lagunares de hierbas marinas y los taludes arrecifales de la mayor parte del área de estudio, están compuestos de carbonatos de origen biogénico y poco sedimento fino silicoclástico (ver Greb et al. 1996), con algunas excepciones dentro del sistema lagunar y desembocaduras de ríos.

A continuación se resumen los resultados para cada una de las islas estudiadas y se inclu- ye observaciones sobre la zona costera adyacente a éstas:

Isla Bastimentos: Esta isla se caracterizó por tener la mayor formación de arrecifes a barlovento (expuestos al oleaje y vientos alisios), pero siendo estas comunidades por lo general de baja diversidad y cobertura coralina. Esta formación arrecifal de tipo franjeante corre paralela a la costa a lo largo del lado norte desde el poblado de Bastimentos hasta Old Point, con pequeñas interrupciones frente a algunas playas (Fig. 1). Hacia el lado nordeste, aproximadamente desde Fish Hole y hasta Old Point, ocurre el mejor desarrollo de la barrera 


\section{CUADRO 4}

Número de especies de los principales táxones animales en 18 arrecifes estudiados en Bocas del Toro, Panamá. Diversidad agrupada en protegidos (PRO), expuestos (EXP) y totales (TOT)

$\begin{array}{lccc}\text { Sitio } & \text { Coral } & \text { Octocoral } & \text { Esponja } \\ 25 & 17 & 6 & 9 \\ 26 & 14 & 2 & 2 \\ 27 & 10 & 3 & 2 \\ 28 & 11 & 2 & 7 \\ 29 & 16 & 1 & 5 \\ 30 & 21 & 1 & 5 \\ 31 & 25 & 7 & 5 \\ 32 & 25 & 2 & 18 \\ 33 & 25 & 11 & 21 \\ 34 & 28 & 10 & 7 \\ 35 & 31 & 2 & 14 \\ 36 & 33 & 7 & 15 \\ 37 & 23 & 8 & 19 \\ 38 & 13 & 11 & 11 \\ 39 & 17 & 8 & 10 \\ 40 & 33 & 3 & 24 \\ 41 & 28 & 13 & 21 \\ 42 & 37 & 17 & 7 \\ \text { EXP } & 35 & 22 & 29 \\ \text { PRO } & 47 & 25 & 37 \\ \text { TOT } & 54 & & 37 \\ & & & \end{array}$

y se forma una amplia laguna entre las playas y la cresta de hasta $150 \mathrm{~m}$ de ancho. La mayor parte de esta barrera se encuentra fuera de los límites del Parque Nacional Marino Isla Bastimentos (PNMIB) (Fig. 1). El lado sur de la isla presenta pocos arrecifes y está formado por muchos cayos de origen arrecifal y cubiertos en su mayoría por manglar. Alrededor de estos cayos, por lo general, se encuentran grandes praderas de hierbas marinas y bajos coralinos someros construídos y dominados por los corales Porites furcata, Agaricia tenuifolia y Millepora alcicornis y M. complanata. Los únicos arrecifes franjeantes, someros e interrumpidos por pequeñas ensenadas de manglar, se observan al sureste de la isla desde Alberry's Creek hasta el cayo German Soldier. De igual forma, se observan gran cantidad de parches arrecifales en este sector de la isla desde Old Point hasta Cayo Crawl (Fig. 1). Se destacan aquí los parches cercanos a Old Point, Alberry's Creek y Cayos Jack Analy, por su extensión y alta diversidad y cobertura de corales, en particular Acropora palmata, A. cervi- cornis y Montastraea annularis (ver arrecife 42). El lado suroeste e interno de la isla (Ensenada Hospital), no presenta casi arrecifes y se desarrollan solamente desde el poblado de Bastimentos y Punta Juan Brown hacia el este y hasta la ensenada cercana a el poblado de Short Cut. El arrecife de Punta J. Brown es el más desarrollado en esta área y se encuentra actualmente afectado por la alta sedimentación proveniente del poblado y alrededores. Algunos parches arrecifales de considerable diversidad y cobertura de coral se encuentran hacia el centro de la Ensenada Hospital y al norte de ésta en algunas de las islas de manglar. La deforestación de la zona costera para prácticas de ganadería y agricultura es notable en ciertas áreas al suroeste de la Isla Bastimentos, entre los asentamientos de Jutica y New Guinea. El manglar ha sido cortado en muchos lugares a lo largo de la costa sur dentro de la ensenada, pero se observa una alta regeneración en muchos de estos parches.

Se describieron seis arrecifes franjeantes a lo largo de la costa $(25-29,33)$ y cinco arrecifes asociados a cayos cercanos o parches de coral $(30-32,37,42)$. Los arrecifes del sector norte son característicos de ambientes de alta energía y presentan un talud con poca pendiente y desarrollado hasta $10 \mathrm{~m}$ de profundidad. Los corales son de crecimiento horizontal, por lo general dominado por las especies Diploria clivosa y Porites astreoides y una alta cobertura de algas coralinas. La mayoría de los arrecifes del norte presentan una cobertura de coral inferior al $10 \%$ y una cobertura de macroalgas que oscila entre 42 y $88 \%$, las más altas del área (Cuadro 2). La diversidad de corales, octocorales y esponjas en el norte es inferior al $50 \%$ de la observada en toda el área (Cuadro 4). Es importante notar que la mayor cobertura de esponjas de todos los arrecifes se encontró en el arrecife de Playa Larga (27) con 18\%, representada principalmente por la esponja perforadora Cliona.

Hacia el lado sur y el lado este de la isla se encuentran mejores arrecifes; la mayor cobertura de coral se encontró en el arrecife 37 $(44 \%)$ en un cayo al extremo sur-oeste de la 

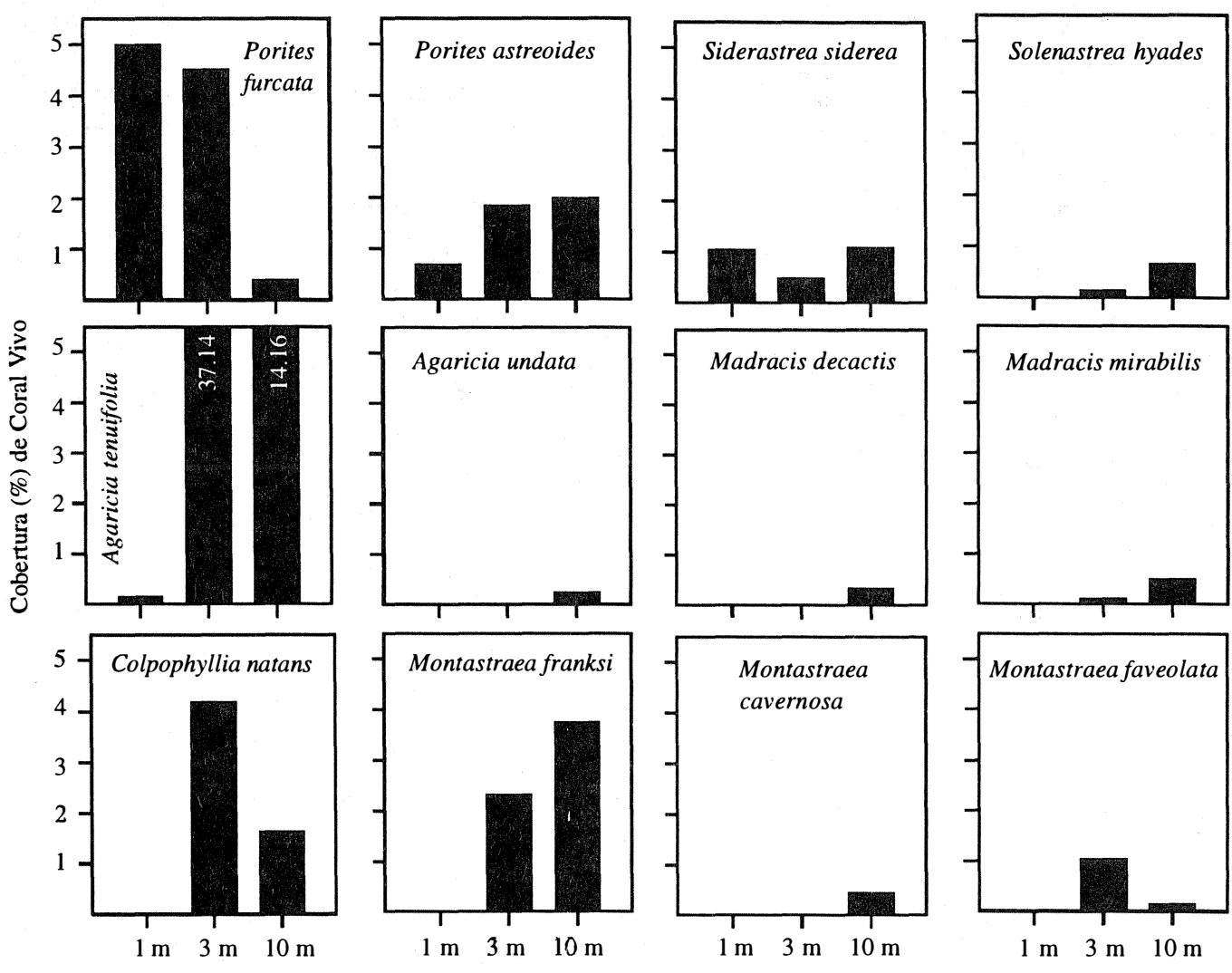

Fig. 6. Cobertura (\%) absoluta de coral vivo para todos los arrecifes agrupados como protegidos (seg ún Fig. 2), para las especie más abundantes de coral encontradas en los transectos y para las tres profundidades (zonación). Se incluyen únicamente las especies con porcentajes mayores al $0.5 \%$.

isla y dentro de los límites del PNMIB. El arrecife Jack Analy (parche 42, Fig. 1) es el más diverso de todos los estudiados con 37 especies de corales y 13 de octocorales y además, la cobertura de corales e hidrocorales es superior al $37 \%$ y $4 \%$, respectivamente (Cuadros 2 y 4 ). Este último grupo es el más abundante con respecto a otras áreas estudiadas. Las esponjas son más diversas y presentan altas coberturas dentro de la Ensenada Hospital al sur de la isla (arrecifes 32 y 33), oscilando entre 18-21 especies y 6-12\% de cobertura (Cuadros 2 y 4). La cobertura de coral en este sector es superior al $25 \%$, y como dato curioso, el parche situado al centro de la ensenada (32) presenta la cobertura más baja de macroalgas (7\%), de todos los arrecifes descritos (Cuadro 2).
Parque Nacional Marino Isla Bastimentos: En general dentro de los límites actuales del área protegida no se encuentra la máxima diversidad de organismos de la región y el desarrollo arrecifal es bajo. Los arrecifes descritos dentro del PNMIB por el lado norte $(27,28$, 30,31 ), presentan una cobertura de coral vivo baja (7-12\%) y una diversidad máxima de 29 especies (Cuadros 2, 4 y 5), la cual representa el 54\% de la diversidad total conocida para Bocas del Toro (Guzmán y Guevara 1998, este estudio). En los Cayos Zapatillas es donde se concentra esa máxima diversidad de corales. El número de especies de esponjas y octocorales es similar e inferior al $28 \%$ del total de especies informadas para las restantes áreas (Cuadros 6 y 7), mientras que la cobertura 


\section{CUADRO 5}

Lista de corales escleractínios encontrados hasta una profundidad de 20 m en cada uno de los 18 arrecifes estudiados en las Islas Colón, Carenero, Bastimentos y Solarte, Provincia de Bocas del Toro, Panamá. Especie presente (x)

Especies

Acropora cervicornis

Acropora palmata

Acropora prolifera

Agaricia agaricites

Agaricia danai

Agaricia fragilis

Agaricia grahamae

Agaricia humilis

Agaricia lamarcki

Agaricia purpurea

Agaricia reticulata

Agaricia tenuifolia

Agaricia undata

Colpophyllia breviserialis

Colpophyllia natans

Dichocoenia stockesi

Diploria clivosa

Diploria labyrinthiformis

Diploria strigosa

Eusmilia fastigiata

Favia fragum

Isophyllastrea rigida

Isophyllia sinuosa

Leptoseris cucullata

Madracis decactis

Madracis mirabilis

Madracis pharensis

Manicina areolata

Meandrina brasiliensis

Meandrina meandrites

Millepora alcicornis

Millepora complanata

Montastraea annularis

Montastraea cavernosa

Montastraea faveolata

Montastraea franksi

Mussa angulosa

Mycetophyllia aliciae

Mycetophyllia danaana

Mycetophyllia ferox

Mycetophyllia lamarckiana

Oculina diffusa

Porites astreoides

Porites colonensis

Porites divaricata

Porites furcata

Porites porites

Scolymia cubensis

Scolymia lacera

Siderastrea radians

Siderastrea siderea

Solenastrea bournoni

Solenastrea hyades

Stephanocoenia intercepta $\begin{array}{llllllllllllllllll}25 & 26 & 27 & 28 & 29 & 30 & 31 & 32 & 33 & 34 & 35 & 36 & 37 & 38 & 39 & 40 & 41 & 42\end{array}$

$\mathrm{X} \quad \mathrm{X} \quad \mathrm{X}$

$\mathrm{X} \quad \mathrm{X}$

$\begin{array}{llll}X & X & X & X \\ & X & & X\end{array}$

$\begin{array}{rrr} & \mathrm{X} & \mathrm{X} \\ & \mathrm{X} & \mathrm{X} \\ \mathrm{X} & \mathrm{X} & \mathrm{X} \\ & & \mathrm{X}\end{array}$

$\begin{array}{ccccc} & \mathrm{X} & \mathrm{X} & & \mathrm{X} \\ \mathrm{X} & \mathrm{X} & & & \mathrm{X} \\ & & \mathrm{X} & \\ & \mathrm{X} & \mathrm{X} & \mathrm{X} & \mathrm{X} \\ & \mathrm{X} & & \mathrm{X} \\ & \mathrm{X} & \mathrm{X} & \\ & & & \mathrm{X}\end{array}$

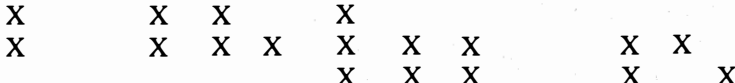

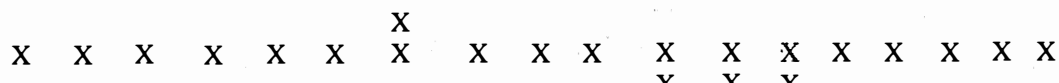

$\mathrm{X}$

$X \quad X \quad X$

$\begin{array}{llllllll} & & & & X & X & X \\ X & X & & & & & X \\ X & X & X & X & X & X & X\end{array}$

$\begin{array}{llllllllllllllllll} & X & X & X & X & X & X & X\end{array}$

$\begin{array}{llllllllll}x & x & x & x & x & x & x & x & x\end{array}$

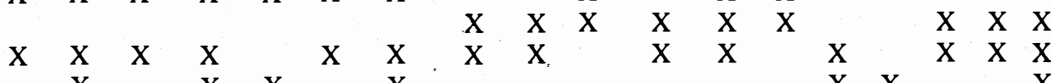

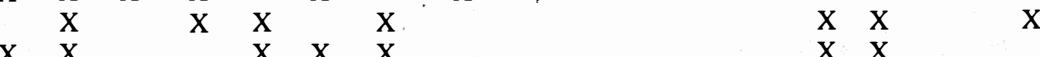

$\begin{array}{lll}X & X & X \\ X & X\end{array}$

$\mathrm{X} \quad \mathrm{X} \quad \mathrm{X}$

$\begin{array}{lllll}\mathrm{X} & \mathrm{X} & \mathrm{X} & \mathrm{X} & \mathrm{X} \\ \mathrm{X} & \mathrm{X} & \mathrm{X} & \mathrm{X} & \mathrm{X}\end{array}$

$X \quad x \quad x$

$\begin{array}{llllllllll}\mathrm{X} & \mathrm{X} & \mathrm{X} & \mathrm{X} & \mathrm{X} & \mathrm{X} & \mathrm{X} & \mathrm{X} & \mathrm{X} & \mathrm{X} \\ \mathrm{X} & \mathrm{X} & \mathrm{X} & \mathrm{X} & & \mathrm{X} & & & \mathrm{X} & \mathrm{X}\end{array}$

$\mathrm{X}$

$\mathrm{X}$

$\mathrm{X}$

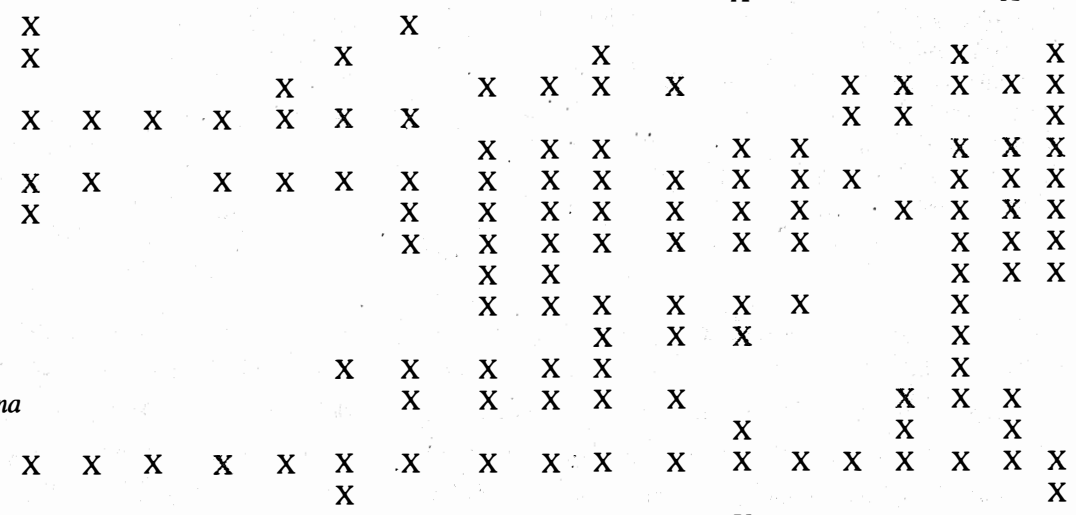

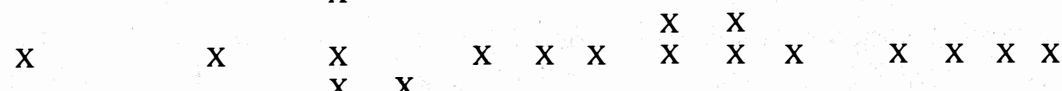

$\begin{array}{lllllllllll}X & X & X & X & X & X & X & X\end{array}$

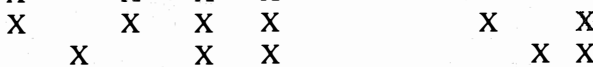

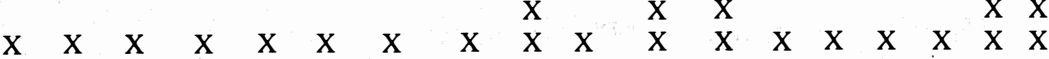

$\mathrm{X} \quad \mathrm{X} \quad \mathrm{X} \quad \mathrm{X}$

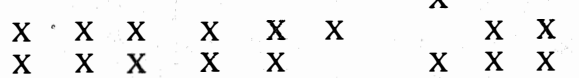




\section{CUADRO 6}

Lista de especies de octocorales, anémonas (*), zoántidos (**) y coral negro (***), encontrados hasta una profundidad de $20 \mathrm{~m}$ en los 18 arrecifes estudiados en las Islas Colón, Carenero, Bastimentos y Solarte, Bocas del Toro, Panamá. Especie presente $(X)$

\begin{tabular}{|c|c|c|c|c|c|c|c|c|c|c|c|c|c|c|c|c|c|c|c|}
\hline Especies & 25 & 26 & 27 & 28 & 29 & 30 & 31 & 32 & 33 & 34 & 35 & 36 & 37 & 38 & 35 & & 0 & 41 & 42 \\
\hline Briareum asbestinum & & & & & & & & & & $X$ & $\mathrm{X}$ & $X$ & $\mathrm{X}$ & & $X$ & & 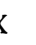 & $\mathrm{X}$ & $X$ \\
\hline Ellisella barbadensis & & & & & & & & & & $\mathrm{X}$ & & & & & & & & & \\
\hline Erythropodium caribaeorum & $\mathrm{X}$ & & & & & & $\mathrm{X}$ & $\mathrm{X}$ & $\mathrm{X}$ & $\mathrm{X}$ & $\mathrm{X}$ & $\mathrm{X}$ & $\mathrm{X}$ & $\mathrm{X}$ & X & & & $\mathrm{X}$ & $\mathrm{X}$ \\
\hline Eunicea calyculata & & & & & & & & & $\mathrm{X}$ & $\mathrm{X}$ & & & K & & & & & & \\
\hline Eunicea fusca & $\mathrm{X}$ & & & & & & & & & & & & $X$ & $\mathrm{X}$ & X & & & & $\mathrm{X}$ \\
\hline Eunicea mammosa & & & & & & & & & & & & & & & $x$ & & & & X \\
\hline Eunicea succinea & & & & & & & $\mathrm{X}$ & & $\mathrm{X}$ & $\mathrm{X}$ & $\mathrm{X}$ & & $\mathrm{X}$ & $\mathrm{X}$ & X & & $\mathrm{X}$ & & $\mathrm{X}$ \\
\hline Eunicea sp. & & & & & & & & & & & & & & & & & & & $x$ \\
\hline Gorgonia flabellum & & & & & & & & & & & & & & & & & & & $x$ \\
\hline Gorgonia ventalina & $\mathrm{X}$ & $\mathrm{X}$ & $\mathrm{X}$ & $\mathrm{X}$ & & & $\mathrm{X}$ & & & $\mathrm{X}$ & & & & & & & & & $\mathrm{x}$ \\
\hline Muriceopsis flavida & $\mathrm{X}$ & $\mathrm{X}$ & $X$ & $\mathrm{X}$ & & & $\mathrm{X}$ & & & & & & & $\mathrm{X}$ & $X$ & & & & \\
\hline Muricea elongata & & : & & & & & & & & & & & & & & & & & \\
\hline Muricea muricata & $X$ & & $\mathrm{X}$ & $\mathrm{X}$ & & & $X$ & & & & & & & $\mathrm{X}$ & & & & & $X$ \\
\hline Nicella schmitti & & & & & $\mathrm{X}$ & . & & & & & & & & & & & & & \\
\hline Plexaura flexuosa & $\mathrm{X}$ & & & & & & $\mathrm{X}$ & & & $\mathrm{X}$ & $\mathrm{X}$ & & & $\mathrm{X}$ & $X$ & & & & $\mathrm{X}$ \\
\hline Plexaura homomalla & & & & & & & & & & & K & & & & & & & & \\
\hline Plexaurella nutans & & & & & & & & & $\mathrm{X}$ & $\mathrm{X}$ & & & & & & & & & $\mathrm{X}$ \\
\hline Plexaurella sp & & & & & & & & $\mathrm{X}$ & & $X$ & & & & & & & $x$ & & \\
\hline Pseudoplexaura sp & & & & & & & & & & & $\mathrm{X}$ & & $X$ & & & & $\mathrm{X}$ & $X$ & $X$ \\
\hline Pseudopterogorgia acerosa & & & & & & & & & $\mathrm{X}$ & $\mathrm{X}$ & & & $X$ & & & & $x$ & & \\
\hline Pseudopterogorgia americanc & & & & & & & & & $\mathrm{X}$ & $X$ & & & & & $x$ & & & $\mathrm{X}$ & \\
\hline Pseudopterogorgia bipinnata & & & & & & & & & & $X$ & & & & $\mathrm{X}$ & $X$ & & & & \\
\hline Pseudopterogorgia sp & & & & & & & & & & & & & & & & & & & \\
\hline Pterogorgia anceps & & & & & & & & & & & $\mathrm{X}$ & & & & & & & & \\
\hline Pterogorgia citrina & & & & & & $\mathrm{X}$ & $X$ & & & & $\mathrm{X}$ & & & $\mathrm{X}$ & & & & & \\
\hline Bartholomea annulata* & & & & $\mathrm{X}$ & & & & & & & & & & & & & & & \\
\hline Bartholomea lucida* & & & & & & & & $\mathrm{X}$ & $\mathrm{X}$ & $X$ & & & $\mathrm{X}$ & & & & $X$ & & \\
\hline Condylactis gigantea* & & & & & & $\mathrm{X}$ & & & & $\mathrm{X}$ & & & & & & & & & I \\
\hline Epicystis crucifer* & & & & & & & & $\mathrm{X}$ & & & & & $\mathrm{X}$ & & & & & & \\
\hline Lebrunia danae* & $\mathrm{X}$ & $\mathrm{X}$ & & & $X$ & $X$ & $X$ & & & & & & $\mathrm{X}$ & & & & & & $X$ \\
\hline Stichodactyla helianthus* & & $\mathrm{X}$ & $X$ & $X$ & & & $X$ & & & & & & & $\mathrm{X}$ & & & & & $X$ \\
\hline Zoanthus pulchellus** & & & & & & & & & & & & $\mathrm{X}$ & & & & & $X$ & & $X$ \\
\hline Palythoa caribaeorum $* *$ & $\mathrm{X}$ & $\mathrm{X}$ & $\mathrm{X}$ & $\mathrm{X}$ & $\mathrm{X}$ & & $\mathrm{X}$ & & $\mathbf{X}$ & & & & $\mathrm{X}$ & $y$ & $\gamma$ & & & & $\mathrm{X}$ \\
\hline $\begin{array}{l}\text { Antipathes } s p^{* * *} \\
\text { Cirrhipathes leutkeni } * * *\end{array}$ & & & & & & & & & $X$ & $X$ & & & & & & & $X$ & $X$ & \\
\hline Cirrhipathes leutkenl & & & & & $X$ & & & & & & & & & & & & & & \\
\hline
\end{tabular}

máxima de estas es de $18 \%$ y $1.4 \%$, respectivamente (Cuadro 2). La cobertura de octocorales en los Cayos Zapatilla podría considerarse alta si se compara con otros arrecifes. La cobertura de macroalgas es alta y osciló entre $45-88 \%$, dominada principalmente por Amphiroa rigida var. antillana. De igual forma, se encontró una alta cobertura (4.3\%) del zoántido Palythoa en los cayos.

Hacia el lado suroeste del PNMIB se describió un solo arrecife (37) representativo, ya que la mayor parte del área está cubierta por cayos de manglar, extensas praderas de hierbas marinas y bajos coralinos de desarrollo limitado y con poca diversidad y cobertura como se mencionó anteriormente. Este arrecife resultó ser el segundo de mayor cobertura de los 18 descritos para esta región (Cuadro 2) y de diversidad de corales intermedia y comparable a la encontrada en los Cayos Zapatillas (Cuadro 4). La diversidad de esponjas y octocorales en este arrecife representan el ca. $51 \%$ y $28 \%$ del total, respectivamente (Cuadros 4-7). La cobertura de macroalgas (ca. 30\%) está entre las más bajas. Los pocos arrecifes dentro de este sector del parque se encuentran localizados alrededor de los cayos Gallego y Canari, al extremo sur del límite.

El reclutamiento de nuevos corales en los arrecifes descritos para el PNMIB (27, 28, 30, 


\section{CUADRO 7}

Lista de especies de esponjas encontradas hasta una profundidad de $20 \mathrm{~m}$ en los 18 arrecifes estudiados en las Islas Colón, Carenero, Bastimentos y Solarte, Provincia de Bocas del Toro, Panamá. Especie presente (X)

\begin{tabular}{|c|c|c|c|c|c|c|c|c|c|c|c|c|c|c|c|c|c|c|}
\hline Especies & 25 & 26 & 27 & 28 & 29 & 30 & 31 & 32 & 33 & 34 & 35 & 36 & 37 & 38 & 39 & 4 & 4 & 142 \\
\hline Amphimedon erina & & & & & & & & $\mathrm{X}$ & & & & & & & & & & \\
\hline Agelas clathrodes & & & & & & & & & & & & & & & & $\mathrm{x}$ & $\gamma$ & \\
\hline Agelas conifera & & & & & & & & $\mathrm{X}$ & $\mathrm{X}$ & $\mathrm{X}$ & $\mathrm{X}$ & & $\mathrm{X}$ & & & & $Y$ & \\
\hline Agelas dispar & & & $\mathrm{X}$ & $\mathrm{X}$ & $\mathrm{X}$ & $\mathrm{X}$ & $\mathrm{X}$ & & $\mathrm{X}$ & & & & & & & & $\gamma$ & \\
\hline Anthosigmella varians & $\mathrm{X}$ & $\mathrm{X}$ & & $\mathrm{X}$ & & $\mathrm{X}$ & $\mathrm{X}$ & & $\mathrm{X}$ & & & $\mathrm{X}$ & & $\mathrm{X}$ & $\mathrm{X}$ & & & \\
\hline Aplysina cauliformis & & & & & & & & & $\mathrm{X}$ & & & & & & $x$ & & $\gamma$ & \\
\hline Aplysina fistularis & $\mathrm{X}$ & & & & & & & $\mathrm{X}$ & $\mathrm{X}$ & & & $\mathrm{X}$ & $\mathrm{X}$ & & & & $Y$ & \\
\hline Aplysina fulva & & & & & & & & $\mathrm{X}$ & & $\mathrm{X}$ & $\mathrm{X}$ & & $\mathrm{X}$ & & $\mathrm{X}$ & & $\gamma$ & \\
\hline Aplysina lacunosa & & & & & & & & & $\mathrm{X}$ & & & $\mathrm{X}$ & $\mathrm{X}$ & & & & 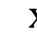 & \\
\hline Artemisina melana & & & & & & & & $\mathrm{X}$ & & & & & & & & & & \\
\hline Callyspongia vaginalis & & & & $\mathrm{X}$ & & & & $\mathrm{X}$ & $\mathrm{X}$ & $\mathrm{X}$ & $\mathrm{X}$ & $\mathrm{X}$ & $\mathrm{X}$ & $\mathrm{X}$ & & & 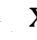 & $\mathrm{X}$ \\
\hline Chondrilla nucula & & & & & $\mathrm{X}$ & & & $\mathrm{X}$ & & & & & & & & & & \\
\hline Cliona delitrix & & & & & & & & $\mathrm{X}$ & $\mathrm{X}$ & & $\mathrm{X}$ & & $\mathrm{X}$ & $\mathrm{X}$ & & & $y$ & \\
\hline Cliona langae & $\mathrm{X}$ & & $\mathrm{X}$ & $\mathrm{X}$ & $\mathrm{X}$ & $\mathrm{X}$ & $\mathrm{X}$ & & $\mathrm{X}$ & & & & & $\mathrm{X}$ & $\mathrm{X}$ & & & \\
\hline Cribrochalina vasculum & & & & & & & & $\mathrm{X}$ & $X$ & & & & & & & & & \\
\hline Ectyoplasia ferox & & & & & & & & & $\mathrm{X}$ & & & & $\mathrm{X}$ & & $x$ & & & \\
\hline Haliclona hogarthi & $\mathrm{X}$ & $\mathrm{X}$ & & & & & & & & & & & & & & & & \\
\hline Ircinia campana & & & & & & & & & $\mathrm{X}$ & $\mathrm{X}$ & $\mathrm{X}$ & X & $\mathrm{X}$ & $\mathrm{X}$ & & & & \\
\hline Ircin & $\mathrm{X}$ & & & & & & & & & & & & & $\mathrm{X}$ & & & & \\
\hline bilina & $\mathrm{X}$ & & & & & & & $\mathrm{X}$ & $\mathrm{X}$ & & $\mathrm{X}$ & & $\mathrm{X}$ & & $\mathrm{X}$ & & & \\
\hline Iotrochota birotulata & $\mathrm{X}$ & & & $\mathrm{X}$ & $\mathrm{X}$ & $\mathrm{X}$ & $\mathrm{X}$ & $\mathrm{X}$ & $\mathrm{X}$ & $\mathrm{X}$ & & $\mathrm{X}$ & $\mathrm{X}$ & $\mathrm{X}$ & $\mathrm{X}$ & & & \\
\hline Monanchora barbadensis & & & & & & & & & & & & $\mathrm{X}$ & & & & & 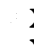 & \\
\hline unguifera & & & & & & & & $\mathrm{X}$ & $\mathrm{X}$ & & 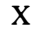 & $\mathrm{X}$ & $\mathrm{X}$ & & & & 2 & \\
\hline Mycale laevis & $\mathrm{X}$ & & & $\mathrm{X}$ & & & $\mathrm{X}$ & $\mathrm{X}$ & & $X$ & $\mathrm{X}$ & $\mathrm{X}$ & & & & & & \\
\hline Neofibularia nolitangere & & & & & & & & & & & $\mathrm{X}$ & $\mathrm{X}$ & $\mathrm{X}$ & & & & & \\
\hline Niphates erecta & & & & & & & & $\mathrm{X}$ & $X$ & $\mathrm{X}$ & $\mathrm{X}$ & $\mathrm{X}$ & $\mathrm{X}$ & $\mathrm{X}$ & & & & \\
\hline Oceanapia bartschi & & & & & & & & & $\mathrm{X}$ & & & & $\mathrm{X}$ & & & & & \\
\hline Petrosia pellasarca & & & & & & & & & & & 2 & & & & & & & \\
\hline igulospiculatus & & & & & & & & $\mathrm{X}$ & & & & & & & $X$ & & & \\
\hline Plakortis halichondroides & & & & & & & & & & & & $\mathrm{X}$ & & & & & & \\
\hline Pseudoceratina crassa & & & & & & & & $\mathrm{X}$ & $\mathrm{X}$ & & $\mathrm{X}$ & $\mathrm{X}$ & $\underset{Y}{X}$ & & $\mathrm{X}$ & & & $x$ \\
\hline Pseudaxinella lunaecharta & & & & & & & & & & & & & $\mathrm{x}$ & $\mathrm{X}$ & & & & \\
\hline Sigmadocia caerulea & & & & & & & & $\mathrm{X}$ & & & & & & & & & & \\
\hline Spheciospongia vesparium & & & & & & & & & & & & & & & & & & \\
\hline Ulosa ruetzleri & $\mathrm{X}$ & & &. $\mathrm{X}$ & $\mathrm{X}$ & $\mathrm{X}$ & $\mathrm{X}$ & $\mathrm{X}$ & $\mathrm{X}$ & & $\boldsymbol{X}$ & $\mathrm{X}$ & $\mathrm{X}$ & $\mathrm{X}$ & & & & \\
\hline Xetospongia muta & & & & & & & & & & & & & & & & & & \\
\hline Xetospongia rosariensis & & & & & & & & & & $\mathrm{X}$ & $\mathrm{X}$ & & $\mathrm{X}$ & & & & 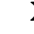 & \\
\hline
\end{tabular}

$31,37)$, es relativamente alto con respecto a otras islas del archipiélago. El sector norte del parque presentó el mayor número de reclutas y de especies, con un total de 0.15-1.1 individuos $/ \mathrm{m}^{2}$, siendo el más alto en los Cayos Zapatillas Oeste (Cuadro 3). Agaricia tenuifolia, Porites astreoides, A. agaricites y Favia fragum son las especies con más reclutas presentes en esta área. Del lado sur del parque se encontró una densidad intermedia de 0.46 individuos $/ \mathrm{m}^{2}$.

Isla Solarte: El mejor desarrollo arrecifal se encuentra hacia los lados noroeste, suroeste y sur, alrededor de los cayos Buttonwood (Fig. 1). La zona costera está siendo deforestada actualmente desde el Gap hasta Punta Hospital por el lado norte y desde esta punta hasta la ensenada Tucker por el lado suroeste. Se observó una alta sedimentación alrededor del sector descrito y sus efectos particularmente en los arrecifes del norte. Se describieron tres arrecifes (34-36), en los alrededores de la isla (Fig. 1). La cobertura de coral vivo y macroalgas es intermedia con respecto a otros arrecifes y oscila entre $27-37 \%$ y $23-51 \%$, respectivamente (Cuadro 2). La mayor cobertura de coral y la menor de algas se observó en el arrecife 36, el 
más alejado de la influencia del área deforestada. La cobertura de los restantes organismos sésiles oscila de baja a intermedia (Cuadro 2). Los arrecifes del lado suroeste presentan una alta cobertura de la macroalga Lobophora variegata, especie de alta capacidad competitiva que dependiendo de su abundancia podría destruir los arrecifes en poco tiempo (De Ruyter van Stevenick et al. 1988, Guzmán obs. per.). El arrecife Solarte Sur (35) presentó una cobertura de esta alga superior al $35 \%$.

La diversidad de corales de los tres arrecifes en conjunto es considerablemente alta con ca. $70 \%$ del total (Cuadro 5) y oscilando para los arrecifes individualmente entre 28-33 especies (Cuadro 4). Las esponjas tienen el 57\% del total (7-15 especies por arrecife), similar al encontrado en Isla Bastimentos (Cuadros 4 y 7). La diversidad de octocorales en conjunto es alta (64\%), siendo los arrecifes 34 y 35 los más diversos con un máximo de 11 especies (Cuadros 4 y 6).

El reclutamiento alrededor de esta isla es similar al encontrado en Isla Bastimentos en cuanto a número de especies, pero intermedio (0.31-0.54 individuos $/ \mathrm{m}^{2}$ ) en cuanto a su densidad (Cuadro 3). Las especies Porites astreoides y $P$. furcata son las más importantes.

Cayo Carenero: Se encuentran arrecifes únicamente en los lados norte (Brown Point) y este (Porvenir) de la isla, donde se forman barreras franjeantes interrumpidas (Fig. 1). El lado sureste de la isla es menos expuesto y afectado por el oleaje, formándose un mejor arrecife a partir de un extenso bajo de octocorales de alta diversidad y cobertura, desde $1.5 \mathrm{~m}$ hasta $5 \mathrm{~m}$ de profundidad, donde se inicia el desarrollo del basamento arrecifal hacia el Canal de Bocas del Toro. Dicho basamento está dominado principalmente por Agaricia tenuifolia y presenta características similares al arrecife de Punta Mängrove (41) en el extremo sureste de la Isla Colón (verr Fig. 1). El lado sur y oeste del cayo está cubierto por praderas de hierbas marinas, principalmente Syringodium filiforme y Thalassia testudinum hasta ca. $2.5 \mathrm{~m}$ de pro- fundidad. Hacia el lado noroeste se encuentra un arrecife prácticamente muerto y cubierto por algas crustosas coralinas, con algunas colonias grandes de Siderastrea siderea del lado protegido de la barrera. Varios parches de arrecife que se extienden hasta los $3 \mathrm{~m}$ de profundidad se encuentran cercanos a la isla y en dirección al centro de la Bahía Sandfly, sin embargo, la diversidad y cobertura de corales en éstos es muy baja. Por lo general están dominados por Millepora spp, y el zoántido Palythoa.

Se describió el arrecife al norte de la isla, Brown Point (38), encontrándose una cobertura de corales de $6 \%$ distribuidos principalmente entre 5-10 m de profundidad y una cobertura de macroalgas de $80 \%$ en todo el arrecife (Cuadro 2, Fig. 3). Curiosamente, el reclutamiento observado es alto con 0.75 individuos $/ \mathrm{m}^{2}$, principalmente Porites astreoides y Favia fragum (Cuadro 3). La diversidad de corales, octocorales y esponjas es bastante baja (Cuadro 4), particularmente los corales con 13 especies que representan el $24 \%$ del total para la región.

Isla Colón: Los arrecifes se encuentran muy bien desarrollados a sotavento de la isla, donde se encuentra una barrera casi continua desde Knapp Hole Point hasta Mangrove Point (Fig. 1). La barrera se interrumpe en la entrada de las bahías y ensenadas de Ground Creek, Conch Point, Big Bight e Istmito, donde dominan praderas de hierbas marinas paralelas a los bosques de manglar. Hacia el lado noroeste, se encuentra una barrera arrecifal en Lime Point que se extiende formando parches coralinos extensos hacia el Canal de Boca del Drago y hacia el norte en Lime Point (Fig. 1). La zona costera en toda la región de Bocas del Drago está siendo deforestada completamente para la construcción de hoteles y los parches de octocorales que ahí abundan al igual que los de Acropora palmata están siendo afectados a consecuencia de la alta sedimentación. Esta deforestación se observa también a lo largo del lado sur. de la isla, particularmente en los alrededores de Big Bight. 
El lado norte y expuesto de la isla no presenta arrecifes franjeantes similares a los de la Isla Bastimentos. La costa desde la Ciudad de Bocas del Toro hasta Big Creek presenta pequeños arrecifes franjeantes discontinuos formados por el coral Millepora complanata y con alta cobertura de coral muerto cubierto por algas coralinas crustosas. Desde este punto hasta el extremo noroeste en Lime Point (Punta Cauro?), que incluye Mimitimbi Bluff, Long Bay Point, Punta Norte y Punta Rocosa, se forma una amplia plataforma coralina discontinua (al frente de playas) y construida principalmente por algas coralinas crustosas y los corales Millepora complanata y Agaricia tenuifolia hasta $4 \mathrm{~m}$ de profundidad y con un ancho variable entre 10-60 m. Estas plataformas se hacen más anchas en las puntas ya mencionadas anteriormente. Entre Lime Point y Punta Norte se encuentra un único parche arrecifal a $300 \mathrm{~m}$ de la costa formado por Acropora palmata, Agaricia tenuifol ia, Siderastrea siderea y el octocoral Muriceopsis flavida, que se extiende hasta los $6 \mathrm{~m}$ de profundidad.

Se describieron tres arrecifes (39-41) alrededor de Isla Colón (Fig. 1). La mayor cobertura de corales de la región se encontró en el arrecife de Mangrove Point (41) con 49\% y una diversidad intermedia de 28 especies (Cuadros 2, 4 y 5). Los otros dos arrecifes presentaron coberturas bajas de coral oscilando entre $1-14 \%$, siendo el arrecife de Lime Point (39) el de menor cobertura de la región y un reflejo claro de la alta sedimentación que ocurre en estos momentos. Sin embargo, en este arrecife se encontraron colonias masivas de Montastraea faveolata y Siderastrea siderea de más de $1.6 \mathrm{~m}$ de alto. La diversidad de corales es más alta en el arrecife de Conch Point (33 especies) y baja en Lime Point (17 especies) (Cuadros 2, 4 y 5). Los parches del coral Acropora cervicornis más extensos encontrados hasta el momento se ubican en los alrededores del arrecife de Conch Point, de 1.5 a $3.5 \mathrm{~m}$ de profundidad.

Los tres arrecifes presentaron baja diversidad de octocorales (3-11 especies), e intermedia de esponjas (10-24). Sin embargo, la cober- tura de octocorales en los arrecifes de Lime Point (39) y Conch Point (40) es la más alta (1.8-3.3\%) encontrada para la región (Cuadros $2,6$ y 7$)$.

La abundancia de corales juveniles (reclutas) es baja en estos tres arrecifes, oscilando de 0-0.21 individuos $/ \mathrm{m}^{2}$ ) (Cuadro 3). Contrario a lo esperado, en el arrecife de Mangrove Point, el de mayor cobertura de coral vivo de la región, no se observó reclutas. Las especies de reclutas más abundantes en estos arrecifes son Favia fragum y Porites astreoides.

Swan Cay (Pájaros): Se hizo un reconocimiento del cayo Swan e islotes Sail y Wreck. No se encontró arrecifes en sus alrededores, pero sí un sustrato consolidado por algas crustosas coralinas típico de hábitats de alta energía. $\mathrm{Al}$ norte de los islotes el desarrollo de corales se limita a colonias de Diploria clivosa creciendo horizontalmente, hasta los $6 \mathrm{~m}$ de profundidad. El lado sur de éstas islas tiene algunos parches pequeños de octocorales, principalmente Muriceopsis flavida, Muricea spp., Pterogorgia anceps y $P$. citrina, rodeados por arena y hierbas marinas hasta $2 \mathrm{~m}$ de profundidad.

\section{DISCUSIÓN}

El presente estudio demuestra la presencia de arrecifes desarrollados y en estado variable de conservación dentro del área insular estudiada del archipiélago de Bocas del Toro. Si se les compara con otros arrecifes de Bocas del Toro, la cobertura promedio de $20 \%$ para coral vivo encontrada en el archipiélago es la mitad de la reportada para los arrecifes continentales de la Ensenada Grande en la Bahía Almirante (Guzmán y Guevara 1998). Comparando con otros arrecifes de Panamá, los arrecifes del sector central del Caribe presentaban para 1991 coberturas de coral de $4 \%$ en la zona industrial de Bahía Las Minas y de 9\% en el sector Portobelo-Isla Grande (Guzmán et al. 1993), con una cobertura de algas superior al $65 \%$ en ambas regiones. Además, Shulman y Robertson (1996), informan de coberturas de $28 \%$ y $26 \%$ para algas y corales, respectivamente, para el sector 
del Porvenir (Sán Blas) en 1990. Sin embargo, para 1992 se informa de una cobertura de algas de entre 55\% y $82 \%$ para la región de San Blas y una cobertura de coral vivo inferior al $10 \%$ en la zona de Porvenir y de $20 \%$ para los $\mathrm{Ca}$ yos Limones y Holandeses (Guzmán en prep.). La cobertura de macroalgas informada en este estudio es alta (50\%), al compararse con los arrecifes continentales cercanos de la Ensenada Grande en Bahía Almirante con 4\% (Guzmán y Guevara 1998).

Los corales encontrados hasta la fecha en Bocas del Toro representan el $88.5 \%$ (54 especies) de la diversidad total informada para el Caribe de Panamá (Holst y Guzmán 1993). Esto significa un aumento de la diversidad para Bocas del Toro de $35 \%$ con respecto al registro previo para arrecifes continentales (Guzmán y Guevara 1998). De igual forma se estima que la diversidad de octocorales se triplicó en el área de estudio, aumentando de ocho a 25 especies y posiblemente representando el 55\% de la diversidad para el resto del país (Guzmán en prep.).

En general, las principales especies formadoras de arrecifes del Caribe están presentes dentro del área de estudio. La ausencia de las especies restantes (11\%), se podría relacionar con el grado de desarrollo de los arrecifes, el cual no se extiende a profunoiidades mayores de $20 \mathrm{~m}$ donde existen hábitats muy específicos, mientras que en otros lugares de Panamá (i.e., San Blas), estos hábitats llegan hasta más de $40 \mathrm{~m}$ de profundidad. Esta limitación está relacionada con la profundidad de penetración de la luz, controlada por factores físico-químicos locales. Entre estos factores los más importantes observados para esta región son la presencia casi permanente de una capa superficial de agua dulce proveniente de la escorrentía en arrecifes protegidos y la alta energía que afecta los arrecifes expuestos del archipiélago. Pocos ríos afectan con sedimentos el área estudiada. La calidad del agua es importante para el desarrollo arrecifal y solamente un número reducido de especies constructoras de arrecifes toleran cambios de salinidad, temperatura y turbidez (Bak 1978,
Rogers 1983, Muthiga y Szmant 1987, Acevedo et al. 1989, Rice y Hunte 1992). Por lo general, la distribución y abundancia de los corales escleractínidos es determinada por el efecto neto de múltiples factores ambientales relacionados entre sí y que pueden actuar de forma aditiva o sinérgica (Tomascik y Sanders 1987).

Se reconoce que dentro del Parque Nacional Marino Isla Bastimentos se encuentran arrecifes con una diversidad inferior de corales escleractínios y organismos asociados si se compara con otras islas cercanas. Además, algunas formaciones arrecifales importantes del sistema insular, donde se observa un mejor estado de conservación y mayor abundancia de organismos asociados al arrecife, no se encuentran dentro del área protegida y requieren de protección inmediata. Tal es el caso del sector este de la Isla Bastimentos, entre Old Point y Crawl Cay, donde se encuentran muchos y extensos parches arrecifales de alta cobertura y diversidad coralina.

Los límites actuales del área protegida (ver Alvarado 1987, Mou-Sue 1993) contemplan una amplia variedad de comunidades costeras, donde se destacan los manglares y praderas de hierbas marinas por su amplia distribución y saludable desarrollo. Los arrecifes coralinos, en particular, se encuentran pobremente representados considerando que el sector norte del parque y similar al resto del archipiélago, es el de mayor superficie marina protegida y donde ocurre la menor abundancia y diversidad de organismos arrecifales. Los arrecifes expuestos descritos en el presente trabajo registraron una cobertura de coral promedio de $8 \%$, comparado con el sector a sotavento del archipiélago donde se registró un promedio de $30 \%$. De los cinco arrecifes descritos dentro del parque (arrecifes 27, 28, 30, 31 y 37), que incluyen la variedad de hábitats del archipiélago, únicamente la mitad de estos presentó una cobertura de corales superior al $10 \%$ y cobertura de macroalgas intermedia (30\%), mientras sólo uno de éstos (Cayo Adriana Este-37) estuvo dentro de los cuatro arrecifes con mayor cobertura dentro del área estudiada 
$(>30 \%)$. Este arrecife se encuentra dentro del sector sur-oeste del parque, protegido de la influencia del oleaje.

Por lo general, el reclutamiento de juveniles en arrecifes del Caribe es lenta (Rylaarsdam 1983, Rogers et al. 1984, Hughes 1985) aun siendo las condiciones favorables, lo cual se debe en gran parte a la biología reproductiva y larval de las principales especies constructoras de arrecifes. Muchas especies importantes como las del complejo Montastraea annularis, Diploria spp., Siderastrea siderea y Acropora palmata presentan fertilización de gametos externa y las larvas se desarrollan por cierto tiempo en la columna de agua, contrario a especies de las familias Agaricidae y Poritidae en las cuales la fertilización e embriogénesis es interna y liberan las larvas (Szmant 1986, Soong 1991). La mayor parte de los reclutas encontrados en la región de Bocas del Toro pertenecen al segundo grupo, en particular las especies Porites astreoides y Favia fragum. Únicamente individuos de una especie perteneciente al primer grupo, S. siderea, reclutó en los arrecifes. Además, la densidad de juveniles y diversidad de especies encontradas para Bocas el Toro es comparable únicamente a los arrecifes con mayor disturbio de Panamá, afectados de la contaminación crónica por petróleo. Para arrecifes de Panamá con nivel intermedio de disturbio (Isla Grande-Portobelo), se ha encontrado un promedio de 4.5 reclu$\mathrm{tas} / \mathrm{m}^{2}$ y una diversidad de hasta 18 especies (Guzmán et al. 1993).

Lo anterior sugiere que el potencial actual del Parque Marino (y otras áreas) en los procesos de regeneración y recuperación de otros arrecifes, dentro y fuera del archipiélago está considerablemente limitado, a pesar de haberse encontrado el mayor número de reclutas $\left(1.1\right.$ individuos $/ \mathrm{m}^{2}$ ) en los arrecifes expuestos del área y en particular en los arrecifes de los Cayos Zapatillas. Basados en el sistema de corrientes costeras del Caribe occidental que afecta al archipiélago y aunque este no se conoce en detalle (pero ver Anónimo 1988b, Guzmán y Jiménez 1992, Greb et al. 1996), se podría especular que muchas de las larvas de organismos constructores de arrecifes se distribuirían en dirección oeste a este, aumentando así el potencial del área de Bocas del Toro como suplidora de material genético al resto de la zona costera de Panamá. Además, este sistema de corrientes proviene del sector norte de Nicaragua y Costa Rica, donde la mayor parte de los arrecifes están deteriorados y afectados por la alta sedimentación (Cortés y Risk 1985) y no se espera una contribución significativa en el proceso de regeneración de arrecifes corriente abajo. Se sugiere que los arrecifes de Bocas del Toro, por encontrarse corriente arriba, podrían suplir áreas alteradas por actividades antrópicas de la zona costera central de Panamá, donde se concentra el mayor desarrollo industrial del Caribe panameño. Sin embargo, el patrón de abundancia y diversidad de reclutas observado dentro del archipiélago no indica que la contribución actualmente sea importante. Es posible que el reclutamiento a sotavento del archipiélago se de a través de larvas producidas localmente (Bahía Almirante y alrededores) y gran parte del reclutamiento a barlovento dependa más de larvas proveniente de arrecifes de otras regiones del Caribe.

En contraste, las diferencias en diversidad de corales y octocorales entre Costa Rica y Panamá son notables; se conocen alrededor de 31 y 26 especies de corales (incluyendo Millepora) y octocorales, respectivamente (Cortés y Guzmán 1985, Guzmán y Cortés 1985, Cortés 1992). Ambos grupos presentan una diversidad ca. 50\% mayor en Panamá, posiblemente debido a la existencia de arrecifes más extensos y hábitats más diversos. Además, es importante señalar que la mayor parte de los arrecifes del Caribe costarricense se encuentran dentro de áreas protegidas, particularmente los arrecifes de Cahuita y Gandoca-Manzanillo, sin embargo, las coberturas de coral vivo máximas son de $11 \%$ y $7 \%$, respectivamente (Cortés y Risk 1985, Cortés 1992, 1994). Lo anteriormente expuesto sirve de argumento para especular sobre la viabilidad de establecer un corredor biológico con normas de manejo conjuntas entre ambos países, cuando realmente no se conocen 
cual es el aporte neto y la procedencia de larvas dentro del archipiélago de Bocas del Toro. Esto, afecta la posibilidad de valorar en términos prácticos el aporte en conservación de arrecifes de las áreas protegidas del PNMIB y del Refugio de Vida Silvestre Gandoca-Manzanillo. No obstante, la relación entre localidades se comprenderá únicamente con estudios de corrientes marinas y genética de las poblaciones de corales, lo cual permitiría establecer criterios científicos para el manejo conjunto de la zona costera. Se requiere de más investigación al respecto.

Finalmente, el conocimiento de los arrecifes de Bocas del Toro ha aumentado notablemente durante el último año y las conclusiones de trabajos anteriores (Greb et al. 1996, Guzmán y Guevara 1998) continúan siendo válidas en términos generales y se resumen a continuación. La información de este estudio permite valorar adecuadamente el estado actual de conservación de los arrecifes de gran parte de Bocas del Toro. Aunque los arrecifes no presentan un atractivo turístico competitivo a nivel del Caribe, cumplen las funciones principales a nivel de ecosistema y sirven de apoyo a poblaciones indígenas locales (Jaén 1993). El desarrollo de esta región traerá como consecuencia inmediata la sobrepoblación en la zona costera, esto se reflejará en la destrucción de manglares y bosques adyacentes, mayor demanda de recursos marinos (ya explotados sin ningún manejo a nivel artesanal), cambios culturales en las poblaciones indígenas quienes tendrán que competir más por espacio y recursos naturales y una disminución en la calidad del agua al aumentar la eutrofización y sedimentación. Actualmente la situación es difícil para las comunidades indígenas locales, quienes ya han comenzado a explotar los recursos del bosque y mar en forma comercial y no a nivel de subsistencia (Jaén 1993). Es notable, que lo anteriormente descrito afectará la productividad y estructura de las comunidades arrecifales, manif estándose en una mortalidad mayor de corales y una baja tasa de reproducción y asentamiento de nuevos corales, efectos que ya han sido documentados ampliamente para otras regiones (Loya 1976, Bak 1978, Cortés y Risk
1985, Tomascick y Sander 1987, Baines y Morrison 1990, Rogers 1990, Goenaga 1991, Tomascik 1991, Wittenberg y Hunte 1992, Guzmán et al. 1993, Guzmán y Holst 1993, Hughes 1994, Roberts 1995). Es posible que los arrecifes del área estudiada ya estén cerca del límite de tolerancia a cambios ambientales; su composición y zonación actual son características que así lo indican. La deforestación indiscriminada en ciertas islas está causando la destrucción acelerada de ảrrecifes, esto sin haberse desarrollado todavía el plan de manejo que integre los recursos naturales de la zona costera y el desarrollo a largo plazo de la región.

\section{RECOMENDACIONES}

Las recomendaciones que se presentan a continuación basadas en el estado de conservación de los arrecifes, están enfocadas hacia las necesidades de mane jo integral de los ecosistemas marinos-terrestres del archipiélago de Bocas del Toro. La visión es amplia y aunque por necesidad se enfatizan las necesidades actuales del Parque Nacional Marino Isla Bastimentos, se pretende extender las recomendaciones a un modelo que incluya los procesos biológicos más importantes en la conservación de arrecifes coralinos. Otras recomendaciones han sido mencionadas anteriormente (Guzmán y Guevara 1998).

(1) Preparar un plan de ordenamiento territorial para uso de la tierra dentro del sector insular de Bocas del Toro, que contemple la protección de bosques y cauces de ríos. Esto podría reducir el impacto e influencia de la sedimentación asociada a la modificación de bosques y cuencas hidrográficas sobre los arrecifes y praderas de hierbas marinas. Ya se observan los efectos de la sedimentación en las Islas Colón, Bastimentos y Solarte.

(2) Estudiar la dinámica y patrones de corrientes marinas que afectan al archipiélago externa e internamente. Esto permitiría comprender con precisión la distribución de los sedimentos acarreados por los ríos y quebradas a lo largo de la costa. Además, se comprendería mejor la distribución de poblaciones y la dis- 
persión de larvas de organismos sésiles que son importantes constructores de arrecifes, al igual que la distribución de propágulos y semillas de manglar y hierbas marinas. Es decir, la identificación de áreas prioritarias de conservación y el potencial de regeneración del sistema, y un aporte a la idea de un corredor marino en la región.

(3) Modificar los límites existentes del PNMIB, con la finalidad de crear nuevas áreas protegidas que incluyan los arrecifes de mayor cobertura y diversidad de especies. Específicamente se recomiendan los siguientes cambios limítrofes: (a) Extender el área terrestre actual para proteger las cuencas de los ríos Sal Creek y Cedar Creek, los cuales drenan hacia el sector oriental de la Isla Bastimentos; (b) Extender los límites terrestres del parque hasta Old Point. Se está desarrollando esta área turísticamente y se espera aumenten los niveles actuales de sedimentación, que afectaría a los arrecifes internos de la laguna oriental y los de $\mathrm{Ca}$ yos Zapatillas; (c) Ampliar los límites marinos del sector oriental de la Isla Bastimentos, desde Old Point hasta Cayo Crawl, donde existen parches arrecifales que hospedan la mayor diversidad y cobertura de corales de este sector, y en particular, las mayores poblaciones de Acropora palmata encontradas hasta el momento en el archipiélago. En Panamá al igual que el resto del Caribe, $A$. palmata sufrió una reducción importante en su cobertura durante la decada de 1980 como consecuencia de enfermedades y el calentamiento de las aguas superficiales (Gladfelter 1982, Guzmán et al. 1991); y (d) Ampliar el límite suroeste del parque hasta los cayos Buttonwood y Punta Hospital, incluyendo una franja terrestre de la Isla Solarte. Esta zona alberga los arrecifes profundos más cercanos al área protegida. Especies importantes de la familia Agaricidae, e.g., Agaricia undata y A. lamarcki, son raras denro del parque debido a que sus hábitats profundos no están bien representados dentro del esquema actual de protección.

(4) Crear pequeñas áreas de conservación bajo un régimen especial de manejo (Reservas
Biológicas), donde se incluyan arrecifes que presentan características únicas dentro del archipiélago que ayudarían eventualmente a preservar la diversidad local y a amortiguar cualquier disminución en la cobertura de otras poblaciones. En particular, se recomienda la creación de una área de reserva en los alrededores de Conch Point, que sirva para proteger las poblaciones existentes del coral Acropora cervicornis, posiblemente las más extensas y someras identificadas hasta el momento, y una especie poco abundante actualmente en el Caribe. De igual forma, se propone la creación de otra reserva en Mangrove Point, la cual presenta actualmente arrecifes en buen estado de conservación. Estos arrecifes, dada su situación de mane jo actual y su localización geográfica entre dos posibles corrientes, tiene el potencial de suplir de larvas al sector este y oeste del archipiélago. Se especula que desde este sector del archipiélago son transportadas las larvas que suplen a los arrecifes de la Ensenada Grande de Almirante.

(5) Decretar períodos de veda para ciertos recursos, principalmente la langosta y el caracol y además, se regulen los diferentes artes de pesca. Promover estudios científicos a nivel de dinámica poblacional (reproducción, reclutamiento, áreas de apareamiento y reproducción, abundancia, distribución) de los principales organismos sujetos a sobrepesca (sensu Tewfik et al. 1998a, b).

\section{AGRADECIMIENTOS}

Agradecemos a Ángel González de PROMAR por su ayuda durante el trabajo de campo y apoyo logístico. A Allen Turner, Eligio Binns y Carlos de Paco por impulsar y apoyar el estudio. A Xenia Guerra por el diseño del mapa y a Karl Kaufmann por preparar la base de datos. A Magnolia Calderón por la revisión completa del manuscrito. Este estudio fue financiado parcialmente por The Nature Conservancy-PROARCA y el Smithsonian Tropical Research Institute. 


\section{RESUMEN}

Se presenta la distribución de arrecifes coralinos a lo largo de la costa de las islas de Bastimentos, Solarte, Carenero y Colón (ca. $120 \mathrm{~km}$ ), Provincia de Bocas del Toro, Panamá Se describe la estructura de 18 arrecifes coralinos, los cuales representan la diversidad de hábitats y condiciones hidrológicas y orográficas de la zona costera del archipiélago. Los arrecifes encontrados en hábitats a barlovento de las islas (expuestos), son menos diversos en corales y presentan una cobertura de coral vivo baja (8\%), mientras que los arrecifes de hábitats protegidos (sotavento), son los de mayor diversidad y cobertura de corales (32\%). Las macroalgas presentaron un patrón similar de cobertura con promedio de $50 \%$, alta si se le compara con arrecifes descritos para la Ensenada Grande de Almirante (4\%), al sur del archipiélago. Se registra un aumento del $35 \%$ en el número de especies de corales para Bocas del Toro; el total de especies conocidas para la región es de 54, lo cual representa el $88 \%$ del total de la diversidad para Panamá. Se encontró que los arrecifes del Parque Nacional Marino Isla Bastimentos no representan la variedad de hábitats, diversidad de especies y abundancia que están presentes en otras localidades fuera de los límites del área protegida. Se señalan recomendaciones para el manejo integral de los recursos naturales marinos y terrestres del archipiélago de Bocas del Toro, donde se proponen variantes a los límites existentes del área protegida y la creación de nuevas categorías de protección (reservas biológicas), entre otros.

\section{REFERENCIAS}

Acevedo, R., J. Morelock \& R.A. Olivieri. 1989. Modification of coral reef zonation by terrigenous sediment stress. Palaios 4: 92-100.

Alvarado, R.H. 1987. Estudio preliminar para el establecimiento del Parque Nacional Marino Isla Bastimentos. INRENARE-CATIE-ANCON, Panamá. 11p.

Anónimo. 1988a. Atlas Nacional de la República de Panamá. Instituto Geográfico Nacional "Tommy Guardia", Panamá. 222 p.

Anónimo. 1988b. Atlas of Pilot Charts for the North Atlantic Ocean. Deffense Mapping Agency, Washington, D.C. $37 \mathrm{p}$.

Baines, G.B.K. \& R.J. Morrison. 1990. Marine pollution from landuse in the Pacific Islands region. Mar. Pollut. Bull. 21 : 506-515.

Bak, R.P.M. 1978. Lethal and sublethal effects of dredging on reef corals. Mar. Pollut. Bull. 9: 14-16.
Cortés, J. 1992. Los arrecifes coralinos del Refugio Nacional de Vida Silvestre Gandoca-Manzanillo, Limón, Costa Rica. Rev. Biol. Trop. 40: 325-333.

Cortés, J. 1994. A reef under siltation stress: a decade of degradation, p. 240-246. In R.N. Ginsburg (ed.). Colloquium on Global Aspects of Coral Reefs: Health, hazards and history, 1993. RSMAS, University of Miami.

Cortés, J. \& H.M. Guzmán. 1985. Organismos de los arrecifes coralinos de Costa Rica III. Descripción y distribución geográfica de los corales pétreos (Anthozoa: Scleractinia) en la costa Caribe. Brenesia 24: 63-123.

Cortés, J. \& M.J. Risk. 1985. A reef under siltation stress: Cahuita, Costa Rica. Bull. Mar. Sci. 36: 339-356.

De Ruyter van Stevenick, E.D., L.L. Van Mulekon \& A.M. Breeman. 1988. Growth inhibition of Lobophora variegata (Lamouroux) Womersley by scleractnian corals. J. Exp. Mar. Biol. Ecol. 115: 169-178.

Gladfelter, W.B. 1982: White-band disease in Acropora palmata: implications for the structure and growth of shallow reefs. Bull. Mar. Sci. 32: 639-643.

Goenaga, C. 1991. The state of coral reefs in the wider Caribbean. Interciencia 16: 12-20.

Greb, L., B. Saric, H. Seyfried, T. Broszonn. S. Brauch, G. Gugau, C. Wiltschko \& R. Leinfelder. 1996. Ökologie ind sedimentologie eines rezenten rampensystem an der Karibikküste von Panama. Profil, Band 10, Universität Stuttgart, 168p.

Guzmán, H.M. \& J. Cortés. 1985. Organismos de los arrecifes coralinos de Costa Rica. IV. Descripción y distribución geográfica de octocorales (Anthozoa: Octocorallia) en la costa Caribe. Brenesia 24: 125-173.

Guzmán, H.M. \& C.E. Jiménez. 1992. Contamination of reef corals by heavy metals along the Caribbean coast of Central America (Costa Rica and Panama). Mar. Pollut. Bull. 24: 554-561.

Guzmán, H.M. \& I. Holst. 1993. Effects of chronic oil-sediment pollution on the reproduction of the Caribbean reef coral Siderastrea siderea. Mar. Pollut. Bull. 26: 276-282.

Guzmán, H.M. \& C. Guevara. 1998. Arrecifes coralinos de Bocas del Toro, Panamá: I. Distribución, estructura y estado de conservación de los arrecifes continentales de la Laguna de Chiriquí y la Bahía de Almirante. Rev. Biol. Trop. 46: 601-623. 
Guzmán, H.M., J.B.C. Jackson \& E. Weil. 1991. Shortterm ecological consequences of a major oil spill on Panamanian subtidal reef corals. Coral Reefs 10: 1-12.

Guzmán, H.M., J.B.C. Jackson \& I. Holst. 1993. Changes and recovery of subtidal reef corals, p. 361-446. In B.D. Keller and J.B.C. Jackson (eds.). Long-term assessment of the oil spill at Bahía Las Minas, Panamá, synthesis Report. U.S. Department of the Interior, Nueva Orleans.

Holst, I. \& H.M. Guzmán. 1993. Lista de corales hermatípicos (Anthozoa: Scleractinia; Hydrozoa: Milleporina) a ambos lados del istmo de Panamá. Rev. Biol. Trop. 41: 535-540.

Hughes, T.P. 1985. Life history and population dynamics of early successional corals. Proc. Fifth Int. Coral Reef Symp. 4: 101-106.

Hughes, T.P. 1994. Catastrophes, phase shifts, and largescale degradation of a Caribbean coral reef. Science 265: $1547-1551$.

Jaén, B.C. 1993. Los pueblos indigenas y el uso de los recursos naturales en Bocas del Toro, p. 119-135. In S. Heckadon-Moreno (ed.). Agenda Ecológica y Social para Bocas del Toro. Continental, Panamá.

Loya, Y.1976. Effects of water turbidity and sedimentation on the community structure of Puerto Rican corals. Bull. Mar. Sci. 26: 450-466.

Moe-Sue, L. 1993. Parque Nacional Marino Isla Bastimentos, p. 163-173. In S. Heckadon-Moreno (ed.). Agenda Ecológica y Social para Bocas del Toro. Continental, Panamá.

Muthiga, A.N. \& A.M. Szmant. 1987. The effects of salinity stress on the rates of aerobic respiration and phosysnthesis in the hermatypic coral Siderastrea siderea. Biol. Bull. 173: 539-551.

Rice, S. A. \& C. L. Hunter. 1992. Effects of suspended sediment and burial on scleractinian corals from West Central Florida patch reefs. Bull. Mar. Sci. 51: 429-442.

Roberts, C.M. 1993. Coral reefs: health, hazards and history. TREE 8: 425-427.

Roberts, C.M. 1995. Effects of fishing on the ecosystem structure of coral reefs. Conserv. Biol. 9: 815-826.

Rodríguez, E., R. Almanza \& R. Alvarado. 1993. Situación biofísica y ambiental de la Provincia de Bocas del Toro, p. 55-72. In S. Heckadon-Moreno (ed.). Agenda
Ecológica y Social para Bocas del Toro. Continental, Panamá.

Rogers, C.S. 1983.Sublethal and lethal effects of sediments applied to common Caribbean reef corals in the field. Mar. Pollut. Bull. 14: 378-382.

Rogers, C.S.1990. Responses of coral reefs and reef organisms to sedimentation. Mar. Ecol. Prog.Ser. 62: 185-202.

Rogers, C.S., H.C Fitz, M.Gilnack, J. Beets \& J. Hardin. 1984. Scleractinian coral recruitment at Salt River submarine canyon, St. Croix, U.S. Virgin Islands. Coral Reefs 3: 69-76.

Rylaarsdam, K.W. 1983. Lif e histories and abundance patterns of colonial corals on Jamaican reefs. Mar. Ecol Prog. Ser. 13: 249-260.

Shulman, M.J. \& D.R. Robertson. 1996. Changes in the coral reefs of San Blas, Caribbean Panama: 19831990. Coral Reefs 15: 231-236.

Soong, K: 1991. Sexual reproductive patterns of shallowwater reef corals in Panama. Bull. Mar. Sci. 49: 832846.

Sullivan, K.M. \& M. Chiappone. 1992. A comparison of belt quadrat and species presence/absence sampling of stony corals (scleractinia and Milleporina) and sponges for evaluating species patterning on patch reefs of the central Bahamas. Bull. Mar. Sci. 50: 464488 .

Sullivan, K.M., M. Chiappone \& C. Lott. 1994. Abundance patterns of stony corals on platform margin reefs of the Caicos Bank. Bahamas J. Sci. 1: 2-12.

Szmant, A.M. 1986. Reproductive ecology of Caribbean reef corals. Coral Reef 5: 43-54.

Tewfik, A., H.M. Guzmán \& G. Jácome. 1998a. Distribution and abundance of the spiny lobster populations (Panulirus argus and P. guttatus) in Cayos Cochinos, Honduras. Rev. Biol. Trop. 46 supl. 4?: 125-136.

Tewfik, A., H.M. Guzmán \& G. Jácome. 1998b. Assessment of the queen conch Strombus gigas (Gastropoda: Strombidae) population in Cayos Cochinos, Honduras. Rev. Biol. Trop. 46 supl. 4?: 137-150.

Tomascik, T. 1991. Settlement patterns of Caribbean scleractinian corals on artificial substrata along a eutrophication gradient, Barbados, West Indies. Mar. Ecol. Prog. Ser., 77: 261-269. 
Tomascik, T. \& F. Sander. 1987. Effects of eutrophication on reef-building corals II. Structure of scleractinians coral communities on fringing reefs, Barbados, West Indies. Mar. Biol. 94: 53-75.
Wittenberg, M. \& W. Hunte. 1992. Effects of eutrophication and sedimentation on juvenile corals. Mar.. Biol. 112:131-138. 